\title{
Fair dynamic valuation of insurance liabilities: A loss averse convex hedging approach
}

\author{
Ze Chen ${ }^{[a] *} \quad$ Bingzheng Chen ${ }^{[b] \dagger}$ \\ Jan Dhaene $e^{[c] \ddagger}$

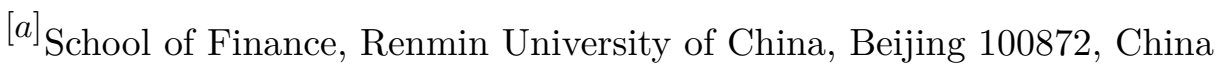 \\ ${ }^{[b]}$ School of Economics and Management, Tsinghua University, Beijing, China \\ ${ }^{[c]}$ Actuarial Research Group, AFI, Faculty of Economics and Business, \\ KU Leuven, Leuven, Belgium
}

Version: January 11, 2020

\begin{abstract}
Hedging techniques have been widely adopted in market-consistent or fair valuation approach required by recent solvency regulations, to take into account the market prices of the hedgeable parts of insurance liabilities. In this study, we investigate the fair dynamic valuation of insurance liabilities, which are model-consistent (mark-to-model), market-consistent (mark-to-market), and time-consistent, as proposed by Barigou et al. (2019) in a multi-period setting. We introduce the loss averse convex hedging technique, which 'punishes' loss outcomes more than gain outcomes. We prove that fair dynamic valuations are equivalent to the class of loss averse convex hedge-based valuation. Moreover, we propose and provide a complete characterization of loss averse mean-variance hedging and show how to implement loss averse mean-variance hedge-based dynamic valuations using numerical examples.
\end{abstract}

Keywords: Fair valuation, loss aversion, convex hedging, market-consistent valuation, safety margins.

\section{Introduction}

Recent solvency regulations for the insurance industry such as Solvency II and the Swiss Solvency Test have required insurance companies to adopt a fair valuation and take into

*zechen@ruc.edu.cn (Corresponding author)

${ }^{\dagger}$ chenbzh@sem.tsinghua.edu.cn

†Jan.Dhaene@kuleuven.be 
account the market prices of the hedgeable parts of insurance liabilities. The fair valuation of insurance liabilities, which are often a combination of hedgeable and unhedgeable risks, equals the sum of the best estimate (hedge) and a risk margin. Under Solvency II, the technical provisions of a fair valuation consist of the best estimate (hedge) and a risk margin. The best estimate (hedge) determines the hedgeable parts of insurance liabilities using the price of its hedge, known as market-consistency. The risk margin is obtained by applying an appropriate risk measure (e.g. the cost-of-capital principle) to the remaining parts after hedging.

Due to the ambiguity in the current regulatory directives on how to determine the hedgeable part of a claim, the calculation of the best estimate is not usually uniquely determined, implying that different hedging techniques are feasible. Dhaene et al. (2017) and Barigou et al. (2019) proposed fair (dynamic) valuation techniques for valuating insurance liabilities in both single-period and multi-period settings and showed that fair valuation is equivalent to a hedge-based valuation approach. Moreover, Dhaene et al. (2017) and Chen et al. (2019) showed that the class of fair (dynamic) valuation is equivalent to convex hedge-based, or CHB, (dynamic) valuations. Specifically, the fair valuation of insurance liabilities follows a two-stage process. In the first step, a convex hedge is set up for the claim to determine its hedgeable part. In the second step, a risk margin is calculated on the basis of the remaining non-hedged part of the claim. The fair value is then the sum of the market value of the hedge and the risk margin value of the residual claim (Figure 1).

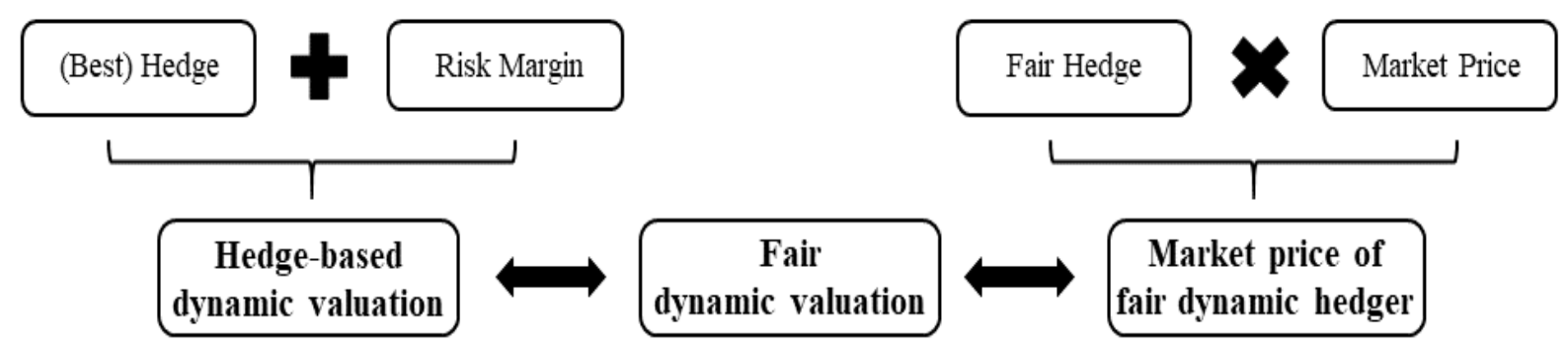

Figure 1: Fair dynamic valuations.

In this study, we extend the work of Barigou et al. (2019) and Chen et al. (2019) by investigating the fair valuation of insurance liabilities using loss averse convex (LAC) hedging, a particular class of convex hedging, in a multi-period setting. The underlying optimization convex function of LAC hedging 'punishes' loss outcomes more than gain outcomes. We further show that the proposed LAC hedging technique tends to 'over-hedge' insurance liabilities to provide an implicit safety margin. The idea behind proposing LAC hedging comes from that any fair (dynamic) valuation is equivalent to the market value of the fair (dynamic) hedging of insurance liabilities (Dhaene et al., 2017, Chen et al., 2019). Thus, a more conservative fair hedging approach can also provide safety margin (or called as prudence margin), that is necessary to ensure that insurers can meet their future obligations. After proposing the LAC hedging approach, we further 
introduce LAC dynamic valuation and prove its equivalence to the class of fair dynamic valuation.

Conventionally, the safety margin of the fair valuation is calculated explicitly using the risk margin. Risk margins are often obtained by applying risk measures to the remaining non-hedgeable risk, such as the widely used cost-of-capital and standard deviation methods. Take mean-variance (MV) hedge-based (MVHB) valuation as an example. Here, the underlying MV hedging approximates the insurance liability using a hedging strategy with a minimal mean squared error (Schweizer, 2010). The MVHB valuation is then obtained by attaching the risk margin to the MV hedge. Similar to the finding of Barigou et al. (2019) that MVHB dynamic valuation is equivalent to the class of fair dynamic valuation, we introduce loss averse MV (LAMV) hedging and prove that LAMV hedge-based (LAMVHB) valuation is also equivalent to fair dynamic valuation. Therefore, any fair dynamic valuation is equivalent to (i.e. can be represented as) MVHB and LAMVHB dynamic valuations. The difference lies in that the safety margin of MVHB valuation only comes from the explicit risk margin after its 'best' hedge, while that of LAMVHB is calculated via both the risk margin and LAMV hedging.

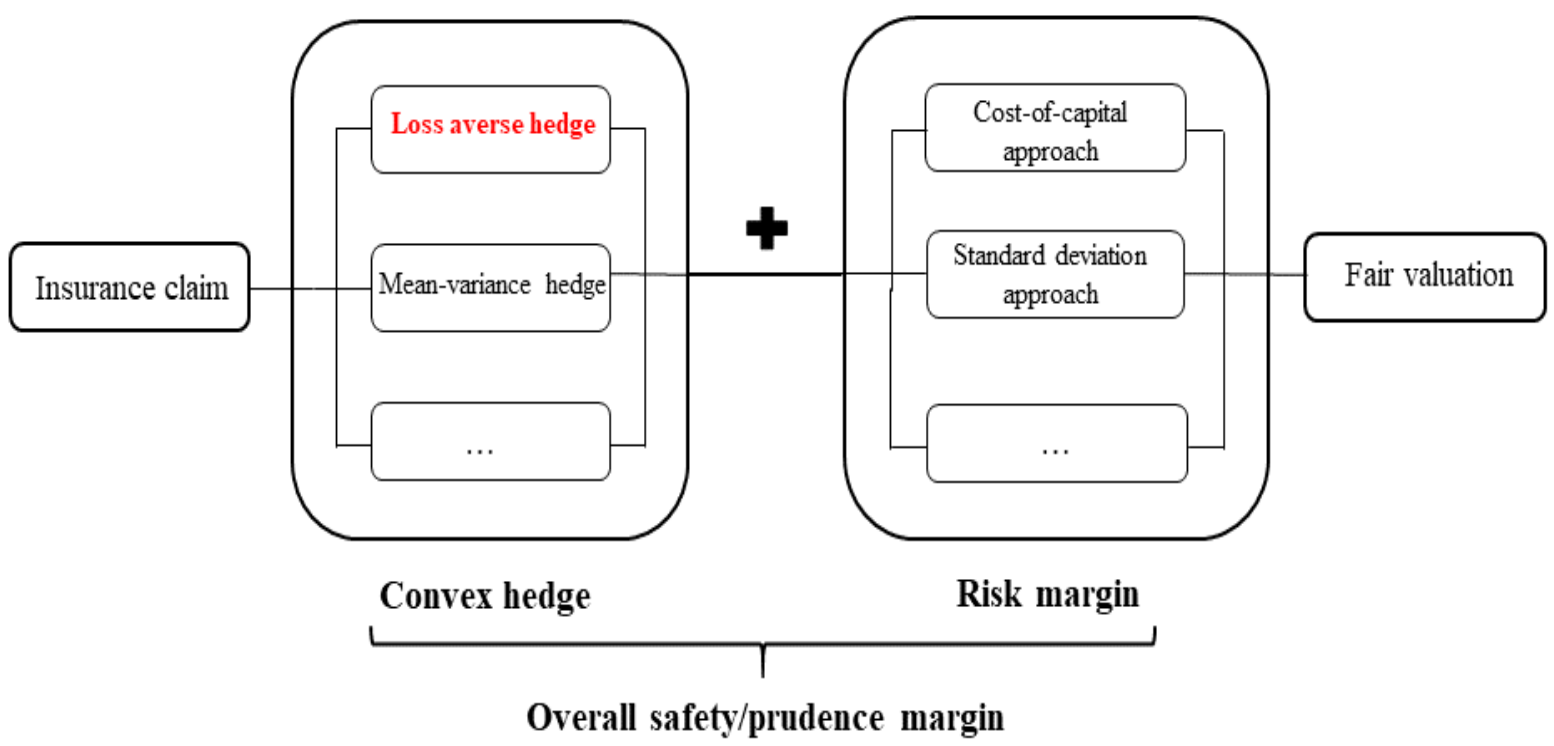

Figure 2: Overall safety/prudence margin of fair/CHB dynamic valuations.

In this paper, we propose a LAC hedging approach to realize the fair dynamic valuation. The LAC hedging approach provides another approach, in addition to the explicit conventional risk margin, to realize the safety margin of fair dynamic valuation implicitly (Figure 2). In particular, we provide a complete characterization of LAMV dynamic hedging and valuation. We further show how to implement fair dynamic valuations using LAMV hedging and provide numerical illustrations.

Our work is related to several streams of the literature. The first stream is related to works on market-consistent and fair valuations of insurance liabilities such as Dhaene 
et al. (2017), Barigou et al. (2019), Chen et al. (2019), Salzmann and Wüthrich (2010), Moehr (2011), Tsanakas et al. (2013), Wüthrich et al. (2013), and Pelsser and Stadje (2014). Another related field is the study of the actuarial valuation principle, including the studies by Kaas et al. (2008) and Norberg (2014). Moreover, time-consistency is an important property of fair dynamic valuations, as shown by the overview by Acciaio and Penner (2011). Lastly, our work is also related to works on thesafety margin of valuation principles, including Christiansen (2013).

The remainder of the paper is structured as follows. In Section 2, we define the general framework of the financial-actuarial world. In Section 3, we revisit the dynamic fair and CHB valuation framework of Chen et al. (2019) and introduce LAC hedge-based (LACHB) and dynamic hedging and valuation. In Section 4, we propose the LAMV dynamic hedge and valuation as well as provide complete characterizations. Section 5 shows fair dynamic valuation using LAMV hedging. Section 6 concludes.

\section{General framework}

We consider a multi-period financial-actuarial world modelled by the probability space $(\Omega, \mathcal{G}, \mathbb{P})$. The current time is 0 and the set of time points $t$ is denoted by $\tau=\{0,1, \ldots, T\}$. The finite and discrete time filtration is $\mathbb{G}=\left\{\mathcal{G}_{t}\right\}_{t \in \tau}$, where $\sigma$-algebra $\mathcal{G}_{t}, t \in \tau$, represents the general information available up to and including time $t$. In this setting, $\mathbb{P}$ is the physical probability measure attaching a probability to any event. Throughout the paper, all the random variables and stochastic processes are defined on this filtered probability space, and all the equalities between the random variables are understood in the $\mathbb{P}$-almost sure sense. We assume that the second moments of all the random variables considered exist under $\mathbb{P}$.

\subsection{Financial market}

We assume $n+1$ non-dividend tradable assets are traded in a deep, liquid, and transparent financial market, which means any tradable asset can be bought or sold at any quantity without transaction costs and other market frictions. In our notation, we denote the market price of risky asset $i$ at time $t \in \tau$ by the notation $Y^{(i)}(t)$. The vector $\boldsymbol{Y}(t)$, $\boldsymbol{Y}(t)=\left(Y^{(0)}(t), Y^{(1)}(t), \ldots, Y^{(n)}(t)\right)$ and $t \in \tau$ arethe time $-t$ prices of all tradable assets. The $(n+1)$-dimensional stochastic process $\boldsymbol{Y}=\{\boldsymbol{Y}(t)\}_{t \in \tau}$ represents the price processes of the traded assets. The price process $\boldsymbol{Y}$ is adapted to the filtration $\mathbb{G}$, which means that

$$
\boldsymbol{Y}(t) \text { is } \mathcal{G}_{t}-\text { measurable, } \quad \text { for any } t=0,1, \ldots, T \text {. }
$$

The filtration $\mathbb{G}$ may simply coincide with the filtration generated by the price process $\boldsymbol{Y}$. However, we consider a more general setting, where $\mathbb{G}$ is not only related to the price history of traded assets, but may also contain additional information on, say, non-tradable claims or a survival index of a particular population. 
A time $-t$ trading strategy (also called a time $-t$ dynamic portfolio), $t \in\{0, \ldots, T-1\}$, is an $(n+1)$-dimensional predictable process $\boldsymbol{\theta}_{t}=\left\{\boldsymbol{\theta}_{t}(u)\right\}_{u \in\{t+1, \ldots, T\}}$ with respect to the filtration $\mathbb{G}$,

$$
\boldsymbol{\theta}_{t}(u) \text { is } \mathcal{G}_{u-1}-\text { measurable, } \quad \text { for any } u=t+1, \ldots, T \text {. }
$$

Thus, a time $-t$ trading strategy is only set up at time $t$ by acquiring a portfolio $\boldsymbol{\theta}_{t}(t+1)$ at that time.

We introduce the notations $\boldsymbol{\theta}_{t}(u)=\left(\theta_{t}^{(0)}(u), \theta_{t}^{(1)}(u) \ldots, \theta_{t}^{(n)}(u)\right)$ for the components of $\boldsymbol{\theta}_{t}(u)$; the quantity $\theta_{t}^{(i)}(u)$ is the number of units invested in asset $i$ in time period $u$, specifically in the time interval $(u-1, u]$. The $\mathcal{G}_{u-1}-$ measurability requirement means that the portfolio composition $\boldsymbol{\theta}_{t}(u)$ for the time period $u$ follows from the general information available up to and including time $u-1$.

At time $t$, the initial investment or endowment of a trading strategy $\boldsymbol{\theta}_{t}$ is given by

$$
\boldsymbol{\theta}_{t}(t+1) \cdot \boldsymbol{Y}(t)=\sum_{i=0}^{n} \theta_{t}^{(i)}(t+1) \times Y^{(i)}(t) .
$$

The value of the trading strategy $\boldsymbol{\theta}_{t}$ at time $u$, just before rebalancing, is expressed as

$$
\boldsymbol{\theta}_{t}(u) \cdot \boldsymbol{Y}(u)=\sum_{i=0}^{n} \theta_{t}^{(i)}(u) \times Y^{(i)}(u), \quad \text { for any } u=t+1, \ldots, T,
$$

whereas its value at time $u$, just after rebalancing, is expressed as

$$
\boldsymbol{\theta}_{t}(u+1) \cdot \boldsymbol{Y}(u)=\sum_{i=0}^{n} \theta_{t}^{(i)}(u+1) \times Y^{(i)}(u), \quad \text { for any } u=t+1, \ldots, T-1 .
$$

Both $\boldsymbol{\theta}_{t}(u) \cdot \boldsymbol{Y}(u)$ and $\boldsymbol{\theta}_{t}(u+1) \cdot \boldsymbol{Y}(u)$ are $\mathcal{G}_{u}$-measurable.

A time $-t$ trading strategy $\boldsymbol{\theta}_{t}$ is said to be self-financing if

$$
\boldsymbol{\theta}_{t}(u) \cdot \boldsymbol{Y}(u)=\boldsymbol{\theta}_{t}(u+1) \cdot \boldsymbol{Y}(u), \quad \text { for any } u=t+1, \ldots, T-1 .
$$

That is, no capital is injected or withdrawn at any rebalancing moment $u=t+1, \ldots, T-1$. Then, the time $-T$ value of any self-financing time $-t$ strategy $\boldsymbol{\theta}_{t}$ can be expressed as

$$
\boldsymbol{\theta}_{t}(T) \cdot \boldsymbol{Y}(T)=\boldsymbol{\theta}_{t}(t+1) \cdot \boldsymbol{Y}(t)+\sum_{u=t+1}^{T} \boldsymbol{\theta}_{t}(u) \cdot \Delta \boldsymbol{Y}(u),
$$

with $\Delta \boldsymbol{Y}(u)=\boldsymbol{Y}(u)-\boldsymbol{Y}(u-1)$. In this formula, $\boldsymbol{\theta}_{t}(u) \cdot \Delta \boldsymbol{Y}(u)$ is the change in the market value of the investment portfolio in the time period $u$, namely between time $u-1$ (just after rebalancing) and time $u$ (just before rebalancing). Hereafter, we denote the set of self-financing time $-t$ trading strategies by $\Theta_{t}$.

Throughout this study, we assume that the market for traded assets is arbitrage-free. That is, there is no self-financing strategy $\boldsymbol{\theta}_{0} \in \Theta_{0}$ such that

$$
\boldsymbol{\theta}_{0}(1) \cdot \boldsymbol{Y}(0)=0, \mathbb{P}\left[\boldsymbol{\theta}_{0}(T) \cdot \boldsymbol{Y}(T) \geq 0\right]=1 \text { and } \mathbb{P}\left[\boldsymbol{\theta}_{0}(T) \cdot \boldsymbol{Y}(T)>0\right]>0 .
$$

In our discrete time setting, the absence of arbitrage is equivalent to the existence of an equivalent martingale measure $\mathbb{Q}^{\mathbb{L}}$, under which the discounted price process $\boldsymbol{Y}$ is a

\footnotetext{
${ }^{1}$ For a proof of this equivalence, see Delbaen and Schachermayer (2006).
} 
$\mathcal{G}$-martingale:

$$
\boldsymbol{Y}(t-1)=\mathbb{E}_{t-1}^{\mathbb{Q}}\left[e^{-\int_{t-1}^{t} r_{s} d s} \boldsymbol{Y}(t)\right], \quad \text { for any } t=1, \ldots, T,
$$

for some (possibly stochastic) interest rate $r_{s}$. In the remainder of the study, we use the notation $\mathbb{E}_{t}^{\mathbb{Q}}[\cdot]:=\mathbb{E}^{\mathbb{Q}}\left[\cdot \mid \mathcal{G}_{t}\right]$. Then, for a time-t self-financing strategy $\boldsymbol{\theta}_{t} \in \Theta_{t}$, from (2) we know its time $-u$ price is given by

$$
\boldsymbol{\theta}_{t}(u+1) \cdot \boldsymbol{Y}(u)=\mathbb{E}_{u}^{\mathbb{Q}}\left[e^{-\int_{u}^{T} r_{s} d s} \boldsymbol{\theta}_{t}(T) \cdot \boldsymbol{Y}(T)\right], \quad \text { for any } u=t, \ldots, T-1 .
$$

Hereafter, the asset 0 is assumed to be the zero-coupon bond paying an amount of 1 at maturity $T$. Its time- $t$ price $B(t, T)$ is given by

$$
Y^{(0)}(t)=B(t, T)=\mathbb{E}_{t}^{\mathbb{Q}}\left[e^{-\int_{t}^{T} r_{s} d s}\right], \quad \text { for any } t=0,1, \ldots, T-1 .
$$

A simple example of a self-financing time $-t$ trading strategy is the static trading strategy $\boldsymbol{\beta}_{t}$ consisting of buying one unit of the zero-coupon bond $B(t, T)$, which pays 1 at $T$, at time $t$ and holding it until maturity $T$. The value of this strategy at time $u$ is given by

$$
\boldsymbol{\beta}_{t}(u) \cdot \boldsymbol{Y}(u)=\mathbb{E}_{u}^{\mathbb{Q}}\left[e^{-\int_{u}^{T} r_{s} d s}\right], \quad \text { for any } u=t+1, \ldots, T .
$$

\section{$2.2 \quad$ Insurance liabilities}

The combined financial actuarial world also hosts a number of insurance liabilities. In our notation, a $t$-claim is payable at time $t$ unless stated otherwise. Furthermore, we denote the set of all $t$-claims defined on $(\Omega, \mathbb{G}, \mathcal{G})$, that is the set of all $\mathcal{G}_{t}$-measurable random variables, by $\mathcal{C}_{t}$. In this study, we consider $T$-claims in most cases, which are payable at time $T$ and belong to the set $\mathcal{C}_{T}$. The notation is generally denoted by $S(T)$ or simply $S$ if no confusion would result. Categorized by whether insurance claims can be hedged by trading strategies, we introduce the notions of two special classes of claims: $t$-hedgeable $T$-claims and $t$-orthogonal $T$-claims.

Definition 1 ( $t$-hedgeable $T$-claim) $A$ t-hedgeable $T$-claim $S^{h}$ is an element of $\mathcal{C}_{T}$ that can be replicated by a time-t self-financing strategy $\boldsymbol{\theta}_{t} \in \Theta_{t}$ :

$$
S^{h}=\boldsymbol{\theta}_{t}(T) \cdot \boldsymbol{Y}(T),
$$

where $\boldsymbol{\theta}_{t}(T) \cdot \boldsymbol{Y}(T)$ is the time-T value of the hedging portfolio $\boldsymbol{\theta}_{t}$.

The set of all time $-t$ hedgeable $T$-claims is $\mathcal{H}_{T}^{t}$. For any time- $t$ hedgeable $T$-claim $S^{h}$, a time- $t$ trading strategy that replicates $S^{h}$ is called a replicating $t-h e d g e$ of $S^{h}$. The time- $t$ price of $S^{h}$ is given by

$$
\boldsymbol{\theta}_{t}(t+1) \cdot \boldsymbol{Y}(t)=\mathbb{E}_{t}^{\mathbb{Q}}\left[e^{-\int_{t}^{T} r_{s} d s} \boldsymbol{\theta}_{t}(T) \cdot \boldsymbol{Y}(T)\right],
$$

where $\mathbb{Q}$ is a generic member of the class of equivalent martingale measures and $\boldsymbol{\theta}_{t}$ is a replicating $t$-hedge of $S^{h}$. 
Definition 2 ( $t$-orthogonal $T$-claim) A t-orthogonal $T$-claim $S^{\perp}$ is an element of $\mathcal{C}_{T}$ that is $\mathbb{P}$-independent of the stochastic process $\mathbf{Y}_{t+1}=\{\boldsymbol{Y}(u)\}_{u \in\{t+1, \ldots, T\}}$ describing the evolution of the traded assets from $t+1$ onwards:

$$
S^{\perp} \perp \mathbf{Y}_{t+1} \text {. }
$$

Hereafter, we denote the set of all $t$-orthogonal $T$-claims by $\mathcal{O}_{T}^{t}$. Both $\mathcal{H}_{T}^{t}$ and $\mathcal{O}_{T}^{t}$ are increasing in $t$. Most $T$-claims are neither $t$-orthogonal nor $t$-hedgeable, but are correlated with the market price of tradable assets.

\subsection{Fair dynamic valuation}

We next revisit the fair dynamic valuations introduced by Barigou et al. (2019) and Chen et al. (2019). In the multi-period setting, a $t$-valuation $\rho_{t}$ assigns to each $T$-claim a $\mathcal{G}_{t}-$ measurable random variable $\rho_{t}[S]$ that represents the $t$-value of the $T$-claim $S$, given the available information at time $t$. A dynamic valuation is a sequence of $t$-valuations 2 .

Definition 3 (t-valuation) At-valuation, $t=0,1, \ldots, T-1$, is a mapping $\rho_{t}: \mathcal{C}_{T} \rightarrow$ $\mathcal{C}_{t}$, attaching a $t$-claim to any $T$-claim $S \in \mathcal{C}_{T}$ :

$$
S \rightarrow \rho_{t}[S],
$$

such that

- $\rho_{t}$ is normalized:

$$
\rho_{t}[0]=0
$$

- $\rho_{t}$ is translation invariant:

$$
\rho_{t}[S+a]=\rho_{t}[S]+B(t, T) a, \quad \text { for any } S \in \mathcal{C}_{T} \text { and } a \in \mathcal{C}_{t} \text { payable at } T .
$$

Definition 4 (Dynamic valuation) A dynamic valuation is a sequence $\left(\rho_{t}\right)_{t=0}^{T-1}$ where for each $t=0,1, \ldots, T-1, \rho_{t}$ is a t-valuation.

Now, we introduce an often used equation. Consider a time $-t$ trading strategy that invests $\rho_{t}[S]$ at time $t$ in the zero-coupon bond $B(t, T)$, for $t=0,1, \ldots, T-1$. The time- $T$ value of this trading strategy $\widetilde{\rho_{t}}$ satisfies that

$$
\widetilde{\rho_{t}}[S]=\frac{\rho_{t}[S]}{B(t, T)} .
$$

The $t$-valuation $\widetilde{\rho_{t}}[S]$ introduced in (4), which corresponds to the value at time $T$ of the investment of the $t$-valuation $\rho_{t}[S]$ in the zero-coupon bond $B(t, T)$, is adopted to compare the $t$-valuations at different times hereafter.

\footnotetext{
${ }^{2}$ See, for instance, Chen et al. (2019), Barigou et al. (2019), Acciaio and Penner (2011), Artzner et al. (2007), and Riedel (2004), who used similar notions.
} 
Table 1 summarizes some important classes of $t$ - and dynamic valuations.

Table 1: Properties of $t$ - and dynamic valuations.

\begin{tabular}{|c|c|c|}
\hline Property & t-valuation $\rho_{t}$ & Dynamic valuation $\left(\rho_{t}\right)_{t=0}^{T-1}$ \\
\hline Actuarial & $\begin{array}{l}\text { If it satisfies } \rho_{t}[S]=B(t, T) \cdot\left(\mathbb{E}^{\mathbb{P}}[S]+R M_{t}[S]\right) \text {, } \\
\text { for any } S \in \mathcal{C}_{T}, \text { where the mapping } R M_{t}: \mathcal{C}_{T} \rightarrow \\
\mathcal{C}_{t} \text { is } \mathbb{P} \text {-law invariant and } \mathbb{P} \text {-independent of time-t } \\
\text { and future asset prices } \mathbf{Y}_{t}=\{\boldsymbol{Y}(u)\}_{u \in\{t, \ldots, T\}} .\end{array}$ & N.A. \\
\hline Model-consistent & $\begin{array}{l}\text { If any } t \text {-orthogonal } T \text {-claim is marked-to-model: } \\
\rho_{t}\left[S^{\perp}\right]=B(t, T) \cdot \mathcal{A}_{t}\left[S^{\perp}\right] \text {, for any } S^{\perp} \in \\
\mathcal{O}_{T}^{t} \text {, where the } t \text {-valuation } \mathcal{A}_{t} \text { is an actuarial } \\
t \text {-valuation. }\end{array}$ & $\begin{array}{l}\text { If any } t \text {-valuation } \rho_{t} \text { is } \\
\text { model-consistent. }\end{array}$ \\
\hline Market-consistent & $\begin{array}{l}\text { If any } t \text {-hedgeable part of any } T \text {-claim is } \\
\text { marked-to-market: } \\
\rho_{t}\left[S+S^{h}\right]=\rho_{t}[S]+\mathbb{E}_{t}^{\mathbb{Q}}\left[e^{-\int_{t}^{T} r_{s} d s} S^{h}\right] \text {, for any } \\
S \in \mathcal{C}_{T} \text { and } S^{h} \in \mathcal{H}_{T}^{t} \text {. }\end{array}$ & $\begin{array}{l}\text { If any } t \text {-valuation } \rho_{t} \text { is } \\
\text { market-consistent. }\end{array}$ \\
\hline Time-consistent & N.A. & $\begin{array}{l}\text { If all } t \text {-valuations in- } \\
\text { volved are connected } \\
\text { in the following way: } \\
\rho_{t}[S]=\rho_{t}\left[\widetilde{\rho}_{t+1}[S]\right] \text {, } \\
\text { for any } S \in \mathcal{C}_{T} \text { and } t= \\
0,1, \ldots, T-2 .\end{array}$ \\
\hline Fair & If it is model- and market-consistent. & $\begin{array}{l}\text { If it is model-, market- } \\
\text { and time-consistent. }\end{array}$ \\
\hline
\end{tabular}

The actuarial and model-consistent properties of $t$ - and dynamic valuations correspond to the traditional valuation of claims in an insurance context. The actuarial condition is a generalization of various insurance premium principles such as the variance principle and standard deviation principle, as discussed by, for example, Bowers (1986), Kaas et al. (2008), and Norberg (2014). One example of actuarial $t$-valuation is the standard deviation principle,

$$
\mathcal{A}_{t}\left[S^{\perp}\right]=B(t, T) \cdot\left(\mathbb{E}_{t}^{\mathbb{P}}\left[S^{\perp}\right]+\alpha \sigma_{t}^{\mathbb{P}}\left[S^{\perp}\right]\right)
$$

with $\sigma_{t}^{\mathbb{P}}\left[S^{\perp}\right]:=\sqrt{\operatorname{Var}^{\mathbb{P}}\left[S^{\perp} \mid \mathcal{G}_{t}\right]}$ and $\alpha>0$. The actuarial $t$-valuation is independent of time- $t$ and future asset prices. The model-consistent condition, defined on the basis of actuarial $t$-valuation, requires that any $t$-orthogonal claim should be valuated by an actuarial $t$-valuation $\mathcal{A}_{t}$ multiplied by the time- $t$ zero-coupon bond price $B(t, T)$. Clearly, the class of actuarial $t$-valuation is a special type of model-consistent $t$-valuation. 
The market-consistent condition extends the notion of cash invariance to all $t$-hedgeable claims by postulating that any $t$-hedgeable claim should be valuated at the price of its replicating $t$-hedge. Some identical or similar conditions can be found in the literature (Kupper et al., 2008, Malamud et al., 2008, Artzner and Eisele, 2010, Pelsser and Stadje, 2014). A financial $t$-valuation is model-consistent, while a model-consistent $t$-valuation is not necessary a financial one. The fair $t$-valuation merges the model- and marketconsistent properties.

The concept of time-consistency, which has been discussed extensively ${ }^{3}$, couples the different static $t$-valuations. Time consistency means that the same time- $t$ value is assigned to a $T$-claim regardless of whether it is calculated in one step or in two steps backwards in time. The fair dynamic valuation merges the properties of model-consistent, market-consistent, and time-consistent valuations (Dhaene et al., 2017, Barigou et al., 2019).

\section{Fair dynamic valuation using LAC hedging}

In this section, we introduce fair dynamic valuation using the LAC hedging approach. We revisit convex hedging and valuation in Section 3.1 and propose the LAC hedging technique in Section 3.2. We show that LAC hedge-based dynamic valuation is equivalent to the class of fair dynamic valuation and provide some examples in Section 3.3.

\subsection{Convex hedging and dynamic valuation}

Dhaene et al. (2017) and Barigou et al. (2019) proved that fair (dynamic) valuation is equivalent to hedge-based valuation in both single-period and multi-period settings. Determining the fair $t$-value of a $T$-claim $S$ at time $t$ departs from splitting the claim into its hedge and the remaining claim:

$$
\rho_{t}[S]=\boldsymbol{\theta}_{t, S}(t+1) \cdot \boldsymbol{Y}(t)+R M_{t}\left[S-\boldsymbol{\theta}_{t, S}(T) \cdot \boldsymbol{Y}(T)\right],
$$

where $\boldsymbol{\theta}_{t, S}$ is a fair $t$-hedge and $R M_{t}$ is a model-dependent valuation. Thus, the fair dynamic valuation approach is hedge-based and it depends on the choice of hedging technique. Dhaene et al. (2017) and Chen et al. (2019) showed that the convex hedging technique is suitable for obtaining the fair valuation of insurance liabilities.

A convex $t$-hedger attaches the hedge $\boldsymbol{\theta}_{S}^{u}$ to any claim $S \in \mathcal{C}_{T}$ such that the claim and time- $T$ value of the hedge are close in the sense that the $\mathbb{P}$-expectation of the $u$-value of their difference is minimized. The choice of the convex function $u$ determines how severe the deviations are punished.

\footnotetext{
${ }^{3}$ See, for example, Cheridito and Kupper (2011). Acciaio and Penner (2011), and Föllmer and Schied (2011) for the discrete time case and Frittelli and Gianin (2004), Delbaen et al. (2010), Pelsser and Stadje (2014), and Feinstein and Rudloff (2015) for the continuous time case.
} 
Definition 5 ( $t$-hedger) At-hedger is a function $\boldsymbol{\theta}_{t}: \mathcal{C}_{T} \rightarrow \Theta_{t}$ that maps any $T$-claim $S$ to a self-financing time-t trading strategy $\boldsymbol{\theta}_{t, S} \in \Theta_{t}$ such that

- $\boldsymbol{\theta}_{t}$ is normalized:

$$
\boldsymbol{\theta}_{t, 0}=\mathbf{0}_{t}
$$

where $\mathbf{0}_{t}$ is the self-financing time-t trading strategy corresponding to the null investment at time $t$, namely $\mathbf{0}_{t}(u)=(0,0, \ldots, 0)$ for all $u=t+1, \ldots, T$.

- $\boldsymbol{\theta}_{t}$ is translation invariant:

$$
\boldsymbol{\theta}_{t, S+a}=\boldsymbol{\theta}_{t, S}+a \boldsymbol{\beta}_{t}, \quad \text { for any } S \in \mathcal{C}_{T} \text { and } a \in \mathcal{C}_{t} \text { payable at } T,
$$

where $\boldsymbol{\beta}_{t}$ is the static trading strategy that consists of buying one unit of the zerocoupon bond $B(t, T)$ and holding it until maturity $T$.

Definition 6 (Convex $t$-hedger) Consider a strictly convex non-negative function $u$ with $u(0)=0$. The $t$-hedger $\theta_{t}^{u}$ determined using

$$
\boldsymbol{\theta}_{t, S}^{u}(t+1)=\arg \min _{\boldsymbol{\mu}_{t} \in \Theta_{t}} \mathbb{E}_{t}^{\mathbb{P}}\left[u\left(\boldsymbol{\mu}_{t}(T) \cdot \boldsymbol{Y}(T)-S\right)\right], \quad \text { for any } S \in \mathcal{C}_{T},
$$

is called a convex t-hedger (with convex function $u$ ).

For any $T$-claim $S$, the self-financing trading strategy $\boldsymbol{\theta}_{t, S}^{u}$ is called a convex $t$-hedge for $S$. The value of the hedge $\boldsymbol{\theta}_{t, S}^{u}$ of $S$ at time $u=t+1, \ldots, T$, before rebalancing, is given by $\boldsymbol{\theta}_{t, S}(u) \cdot \boldsymbol{Y}(u)$, while after rebalancing, it is $\boldsymbol{\theta}_{t, S}^{u}(u+1) \cdot \boldsymbol{Y}(u)$. Hereafter, we introduce the class of convex $t$-valuation based on the convex $t$-hedger.

Chen et al. (2019) introduced the CHB $t$-valuation (dynamic valuation) approach, finding that it is equivalent to fair $t$-valuation (dynamic valuation). In the following paragraph, we revisit the definition of $\mathrm{CHB} t-$ and dynamic valuation.

Definition 7 (CHB $t$ - and dynamic valuation) Consider a $t$-valuation $\rho_{t}: \mathcal{C}_{T} \rightarrow$ $\mathcal{C}_{t}, t=0,1, \ldots, T-1$ and a dynamic valuation $\left(\rho_{t}\right)_{t=0}^{T-1}$.

- $\rho_{t}$ is a CHB t-valuation if there exists a convex $t$-hedger $\boldsymbol{\theta}_{t}^{u}$ and a model-consistent $t$-valuation $\pi_{t}$ such that

$$
\rho_{t}[S]=\boldsymbol{\theta}_{t, S}^{u}(t+1) \cdot \boldsymbol{Y}(t)+\pi_{t}\left[S-\boldsymbol{\theta}_{t, S}^{u}(T) \cdot \boldsymbol{Y}(T)\right]
$$

- $\left(\rho_{t}\right)_{t=0}^{T-1}$ is a CHB dynamic valuation if all $\rho_{t}$ are $C H B$-valuations and connected in the following way:

$$
\rho_{t}[S]=\rho_{t}\left[\widetilde{\rho}_{t+1}[S]\right], \quad \text { for any } S \in \mathcal{C}_{T} \text { and } t=0,1, \ldots, T-2 \text {. }
$$




\subsection{LAC hedging and dynamic valuations}

The convex hedging approach is determined using its underlying convex function $u(x)$. The choice of convex function determines how severe positive and negative deviations (gains and losses) are punished, and hence reflects actuaries' attitude towards hedging outcomes. In this study, we introduce the LAC hedging technique, which differentiates in its attitude towards gains and losses.

Loss aversion, an important concept in decision theory and prospect theory, refers to the fact that a loss of a certain amount leads to losing more satisfaction than the satisfaction brought about from a gain of the equivalent amount. Loss aversion is captured by the following prospect utility, as proposed by Tversky and Kahneman (1992):

$$
u(x)=\left\{\begin{array}{cc}
x^{\alpha}, & x \geq 0 \\
-\lambda(-x)^{\alpha}, & x \leq 0
\end{array} .\right.
$$

Hereafter, we consider a convex hedging technique whose underlying convex function is in line with the loss averse utility form in (7). That is, the underlying convex function displays a higher degree of sensitivity to losses than to gains. We next define the LAC $t$-hedger.

Definition 8 (LAC $t$-hedger) A LACt-hedger $\boldsymbol{\theta}_{t}^{L A C}$ is a convex $t$-hedger determined using

$$
\boldsymbol{\theta}_{t, S}^{L A C}(t+1)=\arg \min _{\boldsymbol{\mu}_{t} \in \Theta_{t}} \mathbb{E}_{t}^{\mathbb{P}}\left[u\left(S-\boldsymbol{\mu}_{t}(T) \cdot \boldsymbol{Y}(T)\right)\right], \quad \text { for any } S \in \mathcal{C}_{T},
$$

and its underlying convex function $u(x)$ satisfies

$$
u(x)=\left\{\begin{array}{cc}
g(x) & x \geqslant 0 \\
\lambda \cdot g(-x) & x<0
\end{array}\right.
$$

where $\lambda>1$ and $g(x) \geqslant 0$ for any $x \geqslant 0$.

In the definition of $u(x)$, the deviation $x$ is a result of the hedging strategy choice in the convex hedging technique in (6). $x$ is a random variable to be observed at time $T$ and its sign is consistent with the deviation of the loss averse utility in (7). Specifically, the $x<0$ cases indicate the losses of insurers, whereas the $x>0$ cases mean the gains. Moreover, the loss aversion degree of a LAC $t$-hedger is measured by the loss aversion coefficient $\lambda$.

As a LAC $t$-hedger's sensitivity to marginal losses and gains is measured by $u^{\prime}(x)$, its coefficient $\lambda$ indicates the ratio of $u(x)$ 's marginal loss sensitivity to its gain sensitivity. We discuss the meaning of the loss aversion coefficient $\lambda$ in the underlying convex function $u(x)$ in the form of equation (7), if we consider $\lambda \geq 0$,

- The convex function $u(x)$ 'punishes' positive deviations more than negative ones when $\lambda<1$; 
- The convex function $u(x)$ 'punishes' negative deviations more than positive ones when $\lambda>1$;

- The convex function $u(x)$ 'punishes' positive and negative deviations similarly when $\lambda=1$.

Since a claim $S$ indicates a liability paying out cashflows, the claim $-S$ is an asset to the insurer that receives the opposite income cashflows. We introduce the notion of the $\mathbb{P}$-symmetric property to measure whether a hedger adopts differentiated assets and liabilities.

Definition 9 A t-hedger $\boldsymbol{\theta}_{t}$ is $\mathbb{P}$-symmetric if

$$
\boldsymbol{\theta}_{t, S}=-\boldsymbol{\theta}_{t,-S}, \quad \text { for any claim } S \in \mathcal{C}_{T} .
$$

A $\mathbb{P}$-symmetric $t$-hedger determines the hedge strategy only depending on the claim's cashflow, regardless of it being a liability or an asset. In other words, a $\mathbb{P}$-symmetric $t$-hedger hedges 'symmetrically' towards a liability (payout cashflows) and an asset with the opposite income cashflows. The following theorem proves that the $\mathbb{P}$-symmetric property is equivalent to that a $t$-hedger 'punishes' positive and negative deviations similarly $(\lambda=1)$.

Theorem 1 The convex t-hedger determined using

$$
\boldsymbol{\theta}_{t, S}^{u}(t+1)=\arg \min _{\boldsymbol{\mu}_{t} \in \Theta_{t}} \mathbb{E}_{t}^{\mathbb{P}}\left[u\left(S-\boldsymbol{\mu}_{t}(T) \cdot \boldsymbol{Y}(T)\right)\right], \quad \text { for any } S \in \mathcal{C}_{T},
$$

with

$$
u(x)=\left\{\begin{array}{cc}
g(x), & x \geqslant 0 \\
\lambda \cdot g(x), & x<0
\end{array} .\right.
$$

is $\mathbb{P}$-symmetric if and only if $\lambda=1$.

Proof: First, we define the following step function:

$$
W(x)=\left\{\begin{array}{ll}
1, & \text { if } x \geqslant 0 \\
\lambda, & \text { if } x<0
\end{array} .\right.
$$

Then, $u(x)=W(x) \cdot g(x)$ and

$$
\frac{d u(x)}{d x}=W(x) \cdot \frac{d g(x)}{d x} .
$$

The first-order conditions of $\mathbb{E}_{t}^{\mathbb{P}}[u(S-\boldsymbol{\mu} \cdot \boldsymbol{Y}(T))]$ are

$$
\mathbb{E}_{t}^{\mathbb{P}}\left[W(x) \cdot \frac{d g(x)}{d x} Y^{(i)}(T)\right]=0, \quad \text { for } i=0,1, . ., n,
$$


where $x=\boldsymbol{\mu} \cdot \boldsymbol{Y}(T)-S$ for any $S \in \mathcal{C}_{T}$. For the special case $g(x)=x^{2}$, equation (9) becomes

$$
\mathbb{E}_{t}^{\mathbb{P}}\left[W(S-\boldsymbol{\mu}(T) \cdot \boldsymbol{Y}(T)) \cdot(S-\boldsymbol{\mu}(T) \cdot \boldsymbol{Y}(T)) Y^{(i)}(T)\right]=0, \text { for } i=0,1, . ., n .
$$

(1) On the one hand, if $\lambda=1$, the convex function $u(x)$ satisfies

$$
u(-x)+u(x)=0 \text {, for any } x \geqslant 0 .
$$

Thus, the convex hedger is $\mathbb{P}$-symmetric, with $\boldsymbol{\theta}_{t, S}^{u}=-\boldsymbol{\theta}_{t,-S}^{u}$ for any $S \in \mathcal{C}_{T}$.

(2) On the other hand, if a convex $t$-hedger $\boldsymbol{\theta}_{t}^{u}$ is $\mathbb{P}$-symmetric, we know $\boldsymbol{\theta}_{t, S}^{u}=\boldsymbol{\theta}_{t,-S}^{u}$ for any $S \in \mathcal{C}_{T}$. Hence, together with the first-order conditions of $\boldsymbol{\theta}_{t, S}^{u}$ and $\boldsymbol{\theta}_{t,-S}^{u}$, we have

$$
\mathbb{E}_{t}^{\mathbb{P}}\left[W\left(S-\boldsymbol{\theta}_{t, S}^{u}(T) \cdot \boldsymbol{Y}(T)\right) \cdot\left(S-\boldsymbol{\theta}_{t, S}^{u}(T) \cdot \boldsymbol{Y}(T)\right) Y^{(i)}(T)\right]=0, \text { for } i=0,1, . ., n,
$$

and

$$
\mathbb{E}_{t}^{\mathbb{P}}\left[W\left(-S+\boldsymbol{\theta}_{t, S}^{u}(T) \cdot \boldsymbol{Y}(T)\right) \cdot\left(S-\boldsymbol{\theta}_{t, S}^{u}(T) \cdot \boldsymbol{Y}(T)\right) Y^{(i)}(T)\right]=0, \text { for } i=0,1, . ., n,
$$

respectively. As equations (11) and (12) hold for any $S \in \mathcal{C}_{T}$ and $\boldsymbol{\theta}_{t, S}^{u}$, the $W(x)$ is an even function with $\lambda=1$.

On the basis of the LAC hedging technique, we introduce the LACHB $t$ - and dynamic valuation.

Definition 10 (LACHB $t$ - and dynamic valuation) Consider a convext-valuation $\rho_{t}: \mathcal{C}_{T} \rightarrow \mathcal{C}_{t}, t=0,1, \ldots, T-1$ and a convex dynamic valuation $\left(\rho_{t}\right)_{t=0}^{T-1}$.

- $\rho_{t}$ is a LACHB $t$-valuation if there exists a LAC $t$-hedger $\boldsymbol{\theta}_{t}^{L A C}$ and a modelconsistent $t$-valuation $\pi_{t}$ such that

$$
\rho_{t}[S]=\boldsymbol{\theta}_{t, S}^{L A C}(t+1) \cdot \boldsymbol{Y}(t)+\pi_{t}\left[S-\boldsymbol{\theta}_{t, S}^{L A C}(T) \cdot \boldsymbol{Y}(T)\right], \quad \text { for any } S \in \mathcal{C}_{T}
$$

- $\left(\rho_{t}\right)_{t=0}^{T-1}$ is a LACHB dynamic valuation if all $\rho_{t}$ are LACHB $t$-valuations.

Chen et al. (2019) showed that the classes of CHB $t$-valuation (dynamic valuation) and fair $t$-valuation (dynamic valuation) are equivalent. As LACHB $t$ - valuations (dynamic valuations) are a particular class of CHB $t$-valuation (dynamic valuation), it is straightforward to show that they are equivalent to the class of fair $t$-valuations (dynamic valuations).

Corollary 1 Consider a t-valuation $\rho_{t}$ and a dynamic valuation $\left(\rho_{t}\right)_{t=0}^{T-1}$ :

- $\rho_{t}$ is a LACHB t-valuation if and only if it is a fair $t$-valuation.

- $\left(\rho_{t}\right)_{t=0}^{T-1}$ is a LACHB dynamic valuation if and only if it is a fair dynamic valuation. 


\subsection{Examples: LAC $t$-hedgers}

We end this section with some examples of LAC $t$-hedgers, whose underlying convex function $u(x)$ is in line with the form in equation (8).

Example 1 Consider a LAC $t$-hedger and its underlying convex function $u(x)$ is

$$
u(x)=\left\{\begin{array}{cc}
e^{\alpha|x|}, & x \geqslant 0 \\
\lambda \cdot e^{\alpha|x|}, & x<0
\end{array}, \lambda>1,\right.
$$

where the function $g(x)$ is an exponential function with $\alpha>0$.

Example 2 Consider a LAC $t$-hedger and a function $g(x)=|x|^{p}, p>1$. Then, its underlying convex function $u(x)$ equals

$$
u(x)=\left\{\begin{array}{cc}
|x|^{p}, & x \geqslant 0 \\
\lambda \cdot|x|^{p}, & x<0
\end{array}, \lambda>1 .\right.
$$

In the following example, we consider a special $u(x)$ in the form of equation (13) with $p=1$, which is a non-strictly convex function, and hence it does not perfectly meet the definition of a LAC $t$-hedger. This example is equivalent to the cost-of-capital risk margin of Solvency II. The cost-of-capital principle is

$$
R M[S]=i_{c o c} \cdot \operatorname{VaR}[S],
$$

where $i_{c o c}$ is the cost-of-capital rate and $p$ is the Value-at-Risk percentile.

Example 3 Consider the non-strictly convex function $g(x)=|x|$; then,

$$
u(x)=\left\{\begin{array}{cc}
|x|, & x \geqslant 0 \\
\lambda \cdot|x|, & x<0
\end{array}, \lambda=\frac{p}{1-p} .\right.
$$

It can be proven that $B(t, T) \cdot V a R_{p}(S)$ is the solution to the following hedger that attaches an investment in the zero-coupon bond to any claim $S$ :

$$
\mu_{t, S}=B(t, T) \cdot \arg \min _{\alpha \in R} \mathbb{E}_{t}^{\mathbb{P}}[u(\alpha-S)], \quad \text { for any } S \in \mathcal{C}_{T} .
$$

The first-order condition is

$$
\begin{aligned}
0 & =\operatorname{Pr}\left(\mu_{t, S} \geq S\right)-\lambda \cdot \operatorname{Pr}\left(\mu_{t, S}<S\right), \\
& =(1+\lambda) \cdot \operatorname{Pr}\left(\mu_{t, S} \geq S\right)-\lambda,
\end{aligned}
$$

implying

$$
\operatorname{Pr}\left(\mu_{t, S} \geq S\right)=\frac{\lambda}{1+\lambda}
$$

and thus,

$$
\mu_{t, S}=B(t, T) \cdot \operatorname{Va} R_{\frac{\lambda}{1+\lambda}}(S)
$$




\section{LAMV hedging}

MV hedging, using the minimal mean squared error, approximates a given payoff using the final value of a trading strategy, as shown by Thomson (2005), Dahl and Møller (2006), and Barigou and Dhaene (2019). The convex function of MV hedging is the quadratic function, and hence MV hedging is a special case of convex hedging. The definition of an MV $t$-hedger is as follows:

Definition 11 (MV t-hedger) The convex $t$-hedger determined using

$\boldsymbol{\theta}_{t, S}^{M V}(t+1)=\arg \min _{\boldsymbol{\mu}_{t} \in \Theta_{t}} \mathbb{E}_{t}^{\mathbb{P}}\left[\left(\boldsymbol{\mu}_{t}(T) \cdot \boldsymbol{Y}(T)-S\right)^{2}\right], \quad$ for any $S \in \mathcal{C}_{T}$ and $t=0,1, \ldots, T-1$,

is called an $M V t$-hedger.

We assume that the time $-T$ value of any time $-t$ trading strategy is square-integrable; hence, a solution to the optimization problem in equation (16) always exists 1 . MV hedging does not differentiate loss and gain deviations. Now, we introduce LAMV hedging, which is more sensitive to losses than gains.

Definition 12 (LAMV t-hedger) The convex $t$-hedger determined using

$$
\boldsymbol{\theta}_{t, S}^{\lambda}(t+1)=\arg \min _{\boldsymbol{\mu}_{t} \in \Theta_{t}} \mathbb{E}_{t}^{\mathbb{P}}\left[u\left(S-\boldsymbol{\mu}_{t}(T) \cdot \boldsymbol{Y}(T)\right)\right], \quad \text { for any } S \in \mathcal{C}_{T},
$$

with

$$
u(x)=\left\{\begin{array}{cc}
x^{2}, & x \geqslant 0 \\
\lambda \cdot x^{2}, & x<0
\end{array}, \lambda>1,\right.
$$

is called an LAMVt-hedger.

Unlike MV hedging, which has an explicit solution and reasonable properties, the LAMV $t$-hedger lacks clear solutions. We follow the literature on asymmetric least squares regression, such as Newey and Powell (1987), Yao and Tong (1996), and Wang et al. (2011), to solve the LAMV $t$-hedger by approximation. Throughout the remainder of this paper, we denote the LAMV $t$-hedger by $\boldsymbol{\theta}_{t}^{\lambda}$ with $\lambda>1$ if no misunderstanding would result. However, in contexts that lack the $\lambda>1$ constraint, we discuss the different ranges of $\lambda$, namely $\lambda>1, \lambda=1$, and $\lambda<1$.

\subsection{LAMV versus MV hedging}

After introducing the LAMV hedging technique, we investigate its hedging properties. We start by comparing the $\mathbb{P}$-expectation property between the LAMV $t$-hedger and MV $t$-hedger.

\footnotetext{
${ }^{4}$ See, for instance, Černỳ and Kallsen (2009).
} 
Theorem 2 The convex t-hedger determined using

$$
\boldsymbol{\theta}_{t, S}^{\lambda}(t+1)=\arg \min _{\boldsymbol{\mu}_{t} \in \Theta_{t}} \mathbb{E}_{t}^{\mathbb{P}}\left[u\left(S-\boldsymbol{\mu}_{t}(T) \cdot \boldsymbol{Y}(T)\right)\right], \quad \text { for any } S \in \mathcal{C}_{T},
$$

with

$$
u(x)=\left\{\begin{array}{cc}
x^{2}, & x \geqslant 0 \\
\lambda \cdot x^{2}, & x<0
\end{array}\right.
$$

satisfies:

(1) If $\lambda>1$,

$$
\mathbb{E}_{t}^{\mathbb{P}}\left[\boldsymbol{\theta}_{t, S}^{\lambda}(T) \cdot \boldsymbol{Y}(T)\right]>\mathbb{E}_{t}^{\mathbb{P}}[S]
$$

(2) If $\lambda=1$,

$$
\mathbb{E}_{t}^{\mathbb{P}}\left[\boldsymbol{\theta}_{t, S}^{\lambda}(T) \cdot \boldsymbol{Y}(T)\right]=\mathbb{E}_{t}^{\mathbb{P}}[S]
$$

(3) If $\lambda<1$,

$$
\mathbb{E}_{t}^{\mathbb{P}}\left[\boldsymbol{\theta}_{t, S}^{\lambda}(T) \cdot \boldsymbol{Y}(T)\right]<\mathbb{E}_{t}^{\mathbb{P}}[S]
$$

Proof: For any $S \in \mathcal{C}_{T}$, the first-order condition of $\boldsymbol{\theta}_{t, S}^{\lambda}$ is $\mathbb{E}_{t}^{\mathbb{P}}\left\{\left[\left(\boldsymbol{\theta}_{t, S}^{\lambda}(T) \cdot \boldsymbol{Y}(T)-S\right) \cdot I_{\boldsymbol{\theta}_{t, S}^{\lambda}(T) \cdot \boldsymbol{Y}(T) \geq S}+\lambda\left(\boldsymbol{\theta}_{t, S}^{\lambda}(T) \cdot \boldsymbol{Y}(T)-S\right) \cdot I_{\boldsymbol{\theta}_{t, S}^{\lambda}(T) \cdot \boldsymbol{Y}(T)<S}\right] Y^{(i)}\right\}=0$, for $i=0,1, . ., n$, where $I_{\{.\}}$is an indicator function. Thus, for the asset $Y^{(0)}$, we have

$$
\mathbb{E}_{t}^{\mathbb{P}}\left[\boldsymbol{\theta}_{t, S}^{\lambda}(T) \cdot \boldsymbol{Y}(T)-S\right]+(\lambda-1) \cdot \mathbb{E}^{\mathbb{P}}\left[\left(\boldsymbol{\theta}_{t, S}^{\lambda}(T) \cdot \boldsymbol{Y}(T)-S\right) \cdot I_{\boldsymbol{\theta}_{t, S}^{\lambda}(T) \cdot \boldsymbol{Y}(T)<S} \mid\right]=0 .
$$

If $\lambda>1$, we have

$$
\mathbb{E}_{t}^{\mathbb{P}}\left[\boldsymbol{\theta}_{t, S}^{\lambda}(T) \cdot \boldsymbol{Y}(T)-S\right]>0,
$$

as

$$
(\lambda-1) \cdot \mathbb{E}_{t}^{\mathbb{P}}\left[\left(\boldsymbol{\theta}_{t, S}^{\lambda}(T) \cdot \boldsymbol{Y}(T)-S\right) \cdot I_{\boldsymbol{\theta}_{t, S}^{\lambda}(T) \cdot \boldsymbol{Y}(T)<S} \mid\right]<0 .
$$

Thus, we have

$$
\mathbb{E}_{t}^{\mathbb{P}}\left[\boldsymbol{\theta}_{t, S}^{\lambda}(T) \cdot \boldsymbol{Y}(T)\right]>\mathbb{E}^{\mathbb{P}}[S] .
$$

The proofs of the $\lambda=1$ and $\lambda<1$ cases are similar and follow from equation (20).

It is straightforward to verify that the MV $t$-hedger $\theta_{t}^{M V}$ corresponds to the convex $t$-hedger in equation (19) with $\lambda=1$. Thus, the MV $t$-hedger has the $\mathbb{P}$-expectation property:

$$
\mathbb{E}_{t}^{\mathbb{P}}[S]=\mathbb{E}_{t}^{\mathbb{P}}\left[\boldsymbol{\theta}_{t, S}^{M V}(T) \cdot \boldsymbol{Y}(T)\right], \quad \text { for any } S \in \mathcal{C}_{T} .
$$

From Lemma 2, we know LAMV $t$-hedgers does not have the $\mathbb{P}$-expectation property. In addition to the $\mathbb{P}$-expectation property, the MV $t$-hedger also has the following propertiest:

$$
\boldsymbol{\theta}_{t, \alpha \times S}^{M V}(T)=\alpha \times \boldsymbol{\theta}_{t, S}^{M V}(T), \quad \text { for any } S \in \mathcal{C}_{T} \text { and scalar } \alpha \geq 0,
$$

and

$$
\boldsymbol{\theta}_{t, S_{1}+S_{2}}^{M V}(T)=\boldsymbol{\theta}_{t, S_{1}}^{M V}+\boldsymbol{\theta}_{t, S_{2}}^{M V}(T), \quad \text { for any } S_{1} \text { and } S_{2} \in \mathcal{C}_{T} .
$$

\footnotetext{
${ }^{5}$ See, for example, Schweizer (2010) and Barigou and Dhaene (2019).
} 
It is straightforward to verify that the LAMV $t$-hedger has the former positive homogeneous property:

$$
\boldsymbol{\theta}_{t, \alpha \times S}^{\lambda}(T)=\alpha \times \boldsymbol{\theta}_{t, S}^{\lambda}(T), \quad \text { for any } S \in \mathcal{C}_{T} \text { and scalar } \alpha \geq 0
$$

However, in general,

$$
\boldsymbol{\theta}_{t, S_{1}+S_{2}}^{\lambda}(T) \neq \boldsymbol{\theta}_{t, S_{1}}^{L A M V}+\boldsymbol{\theta}_{t, S_{2}}^{\lambda}(T)
$$

Proposition 1 For any $S \in \mathcal{C}_{T}$, the convex $t$-hedger defined in equation (19) satisfies

$$
\mathbb{E}_{t}^{\mathbb{P}}\left[\left|\boldsymbol{\theta}_{t, S}^{\lambda}(T) \cdot \boldsymbol{Y}(T)-S\right| \cdot I_{\boldsymbol{\theta}_{t, S}^{\lambda} \cdot \boldsymbol{Y}(T) \geq S}\right]=\frac{\lambda}{\lambda+1} \mathbb{E}_{t}^{\mathbb{P}}\left[\left|\boldsymbol{\theta}_{t, S}^{\lambda}(T) \cdot \boldsymbol{Y}(T)-S\right|\right],
$$

where $\lambda$ is the loss aversion coefficient.

Proof: For any $S \in \mathcal{C}_{T}$, the first-order conditions of $\boldsymbol{\theta}_{t, S}^{\lambda}$ are $\mathbb{E}_{t}^{\mathbb{P}}\left\{\left[\left(\boldsymbol{\theta}_{t, S}^{\lambda}(T) \cdot \boldsymbol{Y}(T)-S\right) \cdot I_{\boldsymbol{\theta}_{t, S}^{\lambda} \cdot \boldsymbol{Y}(T) \geq S}+\lambda\left(\boldsymbol{\theta}_{t, S}^{\lambda}(T) \cdot \boldsymbol{Y}(T)-S\right) \cdot I_{\boldsymbol{\theta}_{t, S}^{\lambda}(T) \cdot \boldsymbol{Y}(T)<S}\right] Y^{(i)}\right\}=0$, for $i=0,1, \ldots n$, where $I_{\{.\}}$is an indicator function. For the asset $Y^{(0)}$, we have

$$
(1+\lambda) \cdot \mathbb{E}_{t}^{\mathbb{P}}\left[\left|\boldsymbol{\theta}_{t, S}^{\lambda}(T) \cdot \boldsymbol{Y}(T)-S\right| \cdot I_{\boldsymbol{\theta}_{t, S}^{\lambda}(T) \cdot \boldsymbol{Y}(T) \geq S}\right]-\lambda \cdot \mathbb{E}_{t}^{\mathbb{P}}\left[\left|\boldsymbol{\theta}_{t, S}^{\lambda}(T) \cdot \boldsymbol{Y}(T)-S\right|\right]=0 .
$$

Subsequently, we have

$$
\frac{\mathbb{E}_{t}^{\mathbb{P}}\left[\left|\boldsymbol{\theta}_{t, S}^{\lambda}(T) \cdot \boldsymbol{Y}(T)-S\right| \cdot I_{\boldsymbol{\theta}_{t, S}^{\lambda}(T) \cdot \boldsymbol{Y}(T) \geq S}\right]}{\mathbb{E}_{t}^{\mathbb{P}}\left[\left|\boldsymbol{\theta}_{t, S}^{\lambda}(T) \cdot \boldsymbol{Y}(T)-S\right|\right]}=\frac{\lambda}{\lambda+1}
$$

Proposition 1 shows that the LAMV $t$-hedger determines the optimal hedging such that the $\frac{\lambda}{\lambda+1}$ ratio of the mean absolute deviations come from the gain deviation cases. For the MV $t$-hedger with $\lambda=1$, the expected deviations that come from the gain and loss scenarios are equal. Given that $\lambda>1$ for the LAMV hedge, more expected deviations come from gains than losses. Thus, the loss aversion coefficient $\lambda$ "controls" the percentage of absolute deviations coming from gains.

\subsection{Gain and loss deviations}

Moreover, in addition to the properties mentioned above, the loss aversion coefficient $\lambda$ makes the LAMV $t$-hedger $\boldsymbol{\theta}_{t}^{\lambda}$ different from the MV $t$-hedger. The following proposition shows that the MV $t$-hedger is $\mathbb{P}$-symmetric, while the LAMV $t$-hedger is not. 
Proposition 2 The convex $t$-hedger determined in equation (19) satisfies:

(1) If $\lambda>1$,

$$
\mathbb{E}_{t}^{\mathbb{P}}\left[\left(\boldsymbol{\theta}_{t, S}^{\lambda}(T)+\boldsymbol{\theta}_{t,-S}^{\lambda}(T)\right) \cdot \boldsymbol{Y}(T)\right]>0 ;
$$

(2) If $\lambda=1$,

$$
\mathbb{E}_{t}^{\mathbb{P}}\left[\left(\boldsymbol{\theta}_{t, S}^{\lambda}(T)+\boldsymbol{\theta}_{t,-S}^{\lambda}(T)\right) \cdot \boldsymbol{Y}(T)\right]=0
$$

(3) If $\lambda<1$,

$$
\mathbb{E}_{t}^{\mathbb{P}}\left[\left(\boldsymbol{\theta}_{t, S}^{\lambda}(T)+\boldsymbol{\theta}_{t,-S}^{\lambda}(T)\right) \cdot \boldsymbol{Y}(T)\right]<0
$$

Proof: For any $S \in \mathcal{C}_{T}$, the first-order condition of $\boldsymbol{\theta}_{t, S}^{\lambda}$ with respect to the asset $Y^{(0)}$ is derived in equation (20). This is equivalent to

$$
\mathbb{E}_{t}^{\mathbb{P}}\left[\boldsymbol{\theta}_{t, S}^{\lambda}(T) \cdot \boldsymbol{Y}(T)-S\right]=(\lambda-1) \cdot \mathbb{E}_{t}^{\mathbb{P}}\left[\left(S-\boldsymbol{\theta}_{t, S}^{\lambda}(T) \cdot \boldsymbol{Y}(T)\right) \cdot I_{\boldsymbol{\theta}_{t, S}^{\lambda}} \cdot \boldsymbol{Y}(T)<S \mid\right] .
$$

From the proof of Proposition refprop2, for any $S \in \mathcal{C}_{T}$, we know

$$
\mathbb{E}_{t}^{\mathbb{P}}\left[\left(S-\boldsymbol{\theta}_{t, S}^{\lambda}(T) \cdot \boldsymbol{Y}(T)\right) \cdot I_{\boldsymbol{\theta}_{t, S}^{\lambda}(T) \cdot \boldsymbol{Y}(T)<S} \mid\right]=\frac{1}{\lambda+1} \mathbb{E}_{t}^{\mathbb{P}}\left[\left|\boldsymbol{\theta}_{t, S}^{\lambda}(T) \cdot \boldsymbol{Y}(T)-S\right|\right] .
$$

Combining equations (25) and (26), we have

$$
\mathbb{E}_{t}^{\mathbb{P}}\left[\boldsymbol{\theta}_{t, S}^{\lambda}(T) \cdot \boldsymbol{Y}(T)-S\right]=\frac{\lambda-1}{\lambda+1} \mathbb{E}_{t}^{\mathbb{P}}\left[\left|\boldsymbol{\theta}_{t, S}^{\lambda}(T) \cdot \boldsymbol{Y}(T)-S\right|\right],
$$

for any $S \in \mathcal{C}_{T}$. Then, for the claim $-S$, we have

$$
\mathbb{E}_{t}^{\mathbb{P}}\left[\boldsymbol{\theta}_{t,-S}^{\lambda}(T) \cdot \boldsymbol{Y}(T)-(-S)\right]=\frac{\lambda-1}{\lambda+1} \mathbb{E}_{t}^{\mathbb{P}}\left[\left|\boldsymbol{\theta}_{t,-S}^{\lambda}(T) \cdot \boldsymbol{Y}(T)-(-S)\right|\right] .
$$

Adding these two equations leads to

$$
\begin{aligned}
& \mathbb{E}_{t}^{\mathbb{P}}\left[\left(\boldsymbol{\theta}_{t, S}^{\lambda}(T) \cdot \boldsymbol{Y}(T)+\boldsymbol{\theta}_{t,-S}^{\lambda}(T)\right) \cdot \boldsymbol{Y}(T)\right] \\
& =\mathbb{E}_{t}^{\mathbb{P}}\left[\left(\boldsymbol{\theta}_{t, S}^{\lambda}(T) \cdot \boldsymbol{Y}(T)-S+\boldsymbol{\theta}_{t,-S}^{\lambda}(T)\right) \cdot \boldsymbol{Y}(T)+S\right] \\
& =\mathbb{E}_{t}^{\mathbb{P}}\left[\boldsymbol{\theta}_{t, S}^{\lambda}(T) \cdot \boldsymbol{Y}(T)-S\right]+\mathbb{E}_{t}^{\mathbb{P}}\left[\boldsymbol{\theta}_{t,-S}^{\lambda}(T) \cdot \boldsymbol{Y}(T)-(-S)\right] \\
& =\frac{\lambda-1}{\lambda+1} \mathbb{E}_{t}^{\mathbb{P}}\left[\boldsymbol{\theta}_{t, S}^{\lambda}(T) \cdot \boldsymbol{Y}(T)-(-S)|+| \boldsymbol{\theta}_{t,-S}^{\lambda}(T) \cdot \boldsymbol{Y}(T)-(-S) \mid\right],
\end{aligned}
$$

for any $S \in \mathcal{C}_{T}$. Since both $\left|\boldsymbol{\theta}_{t, S}^{\lambda}(T) \cdot \boldsymbol{Y}(T)-(-S)\right|$ and $\left|\boldsymbol{\theta}_{t, S}^{\lambda}(T) \cdot \boldsymbol{Y}(T)-(-S)\right|$ are positive, the positiveness or negativeness depends on the value of $\lambda$. Thus, we can draw the conclusions in equations (22), (23), and (24).

Proposition 1 implies that the MV $t$-hedger is $\mathbb{P}$-symmetric. Since the convex $t$-hedger $\boldsymbol{\theta}_{t, S}^{\lambda}$ satisfies $\mathbb{E}_{t}^{\mathbb{P}}\left[\left(\boldsymbol{\theta}_{t, S}^{\lambda}+\boldsymbol{\theta}_{t,-S}^{\lambda}\right) \cdot \boldsymbol{Y}(T)\right]>0$ if $\lambda>1$, it is easy to verify that the LAMV $t$-hedger is not $\mathbb{P}_{\text {-symmetric. }}$ 
Hereafter, we analyse the effects of the loss aversion coefficient $\lambda$ in an LAMV $t$-hedger. We start by decomposing the expected absolute value of an LAMV $t$-hedger's deviation. The expected absolute deviation $\mathbb{E}_{t}^{\mathbb{P}}\left[\boldsymbol{\theta}_{t, S}^{\lambda} \cdot \boldsymbol{Y}(T)-S \mid\right]$ can be decomposed into

$$
\mathbb{E}_{t}^{\mathbb{P}}\left[\left|\boldsymbol{\theta}_{t, S}^{\lambda}(T) \cdot \boldsymbol{Y}(T)-S\right| \cdot I_{\boldsymbol{\theta}_{t, S}^{\lambda}(T) \cdot \boldsymbol{Y}(T) \geq S}\right]+\mathbb{E}_{t}^{\mathbb{P}}\left[\left|\boldsymbol{\theta}_{t, S}^{\lambda}(T) \cdot \boldsymbol{Y}(T)-S\right| \cdot I_{\boldsymbol{\theta}_{t, S}^{\lambda}(T) \cdot \boldsymbol{Y}(T)<S}\right],
$$

where $I_{\{.\}}$is an indicator function. We adopt

$$
\mathbb{E}_{t, g a i n}^{\mathbb{P}}\left[\left|\boldsymbol{\theta}_{t, S}^{\lambda}(T) \cdot \boldsymbol{Y}(T)-S\right|\right]=\mathbb{E}_{t}^{\mathbb{P}}\left[\left|\boldsymbol{\theta}_{t, S}^{\lambda}(T) \cdot \boldsymbol{Y}(T)-S\right| \cdot I_{\boldsymbol{\theta}_{t, S}^{\lambda}(T) \cdot \boldsymbol{Y}(T) \geq S}\right],
$$

and

$$
\mathbb{E}_{t, l o s s}^{\mathbb{P}}\left[\left|\boldsymbol{\theta}_{t, S}^{\lambda}(T) \cdot \boldsymbol{Y}(T)-S\right|\right]=\mathbb{E}_{t}^{\mathbb{P}}\left[\left|\boldsymbol{\theta}_{t, S}^{\lambda}(T) \cdot \boldsymbol{Y}(T)-S\right| \cdot I_{\boldsymbol{\theta}_{t, S}^{\lambda}(T) \cdot \boldsymbol{Y}(T) \geq S}\right],
$$

to simplify the notation.

Proposition 3 For any $S \in \mathcal{C}_{T}$, the LAMV hedger $\boldsymbol{\theta}_{t, S}^{\lambda}$ satisfies

$$
\mathbb{E}_{t}^{\mathbb{P}}\left[\boldsymbol{\theta}_{t, S}^{\lambda}(T) \cdot \boldsymbol{Y}(T)\right]-\mathbb{E}_{t}^{\mathbb{P}}[S]=\mathbb{E}_{t, \text { gain }}^{\mathbb{P}}\left[\left|\boldsymbol{\theta}_{t, S}^{\lambda}(T) \cdot \boldsymbol{Y}(T)-S\right|\right]-\mathbb{E}_{t, l o s s}^{\mathbb{P}}\left[\left|\boldsymbol{\theta}_{t, S}^{\lambda}(T) \cdot \boldsymbol{Y}(T)-S\right|\right]
$$

Proof: For any $S \in \mathcal{C}_{T}$, the first-order conditions of $\boldsymbol{\theta}_{t, S}^{\lambda}$ imply equation (27). From Proposition 1, we know that

$$
\mathbb{E}_{t}^{\mathbb{P}}\left[\left(S-\boldsymbol{\theta}_{t, S}^{\lambda}(T) \cdot \boldsymbol{Y}(T)\right) \cdot I_{\boldsymbol{\theta}_{t, S}^{\lambda}(T) \cdot \boldsymbol{Y}(T) \geq S} \mid\right]=\frac{\lambda}{\lambda+1} \mathbb{E}_{t}^{\mathbb{P}}\left[\left|\boldsymbol{\theta}_{t, S}^{\lambda}(T) \cdot \boldsymbol{Y}(T)-S\right|\right],
$$

and

$$
\mathbb{E}_{t}^{\mathbb{P}}\left[\left(S-\boldsymbol{\theta}_{t, S}^{\lambda}(T) \cdot \boldsymbol{Y}(T)\right) \cdot I_{\boldsymbol{\theta}_{t, S}^{\lambda}(T) \cdot \boldsymbol{Y}(T)<S} \mid\right]=\frac{1}{\lambda+1} \mathbb{E}_{t}^{\mathbb{P}}\left[\left|\boldsymbol{\theta}_{t, S}^{\lambda}(T) \cdot \boldsymbol{Y}(T)-S\right|\right]
$$

Then, we have

$$
\begin{gathered}
\mathbb{E}_{t}^{\mathbb{P}}\left[\boldsymbol{\theta}_{t, S}^{\lambda}(T) \cdot \boldsymbol{Y}(T)-S\right]=\frac{\lambda-1}{\lambda+1} \mathbb{E}_{t}^{\mathbb{P}}\left[\left|\boldsymbol{\theta}_{t, S}^{\lambda}(T) \cdot \boldsymbol{Y}(T)-S\right|\right] \\
=\frac{\lambda}{\lambda+1} \mathbb{E}_{t}^{\mathbb{P}}\left[\left|\boldsymbol{\theta}_{t, S}^{\lambda}(T) \cdot \boldsymbol{Y}(T)-S\right|\right]-\frac{1}{\lambda+1} \mathbb{E}_{t}^{\mathbb{P}}\left[\left|\boldsymbol{\theta}_{t, S}^{\lambda}(T) \cdot \boldsymbol{Y}(T)-S\right|\right] \\
=\mathbb{E}_{t}^{\mathbb{P}}\left[\left|\boldsymbol{\theta}_{t, S}^{\lambda}(T) \cdot \boldsymbol{Y}(T)-S\right| \cdot I_{\boldsymbol{\theta}_{t, S}^{\lambda}(T) \cdot \boldsymbol{Y}(T) \geq S}\right]-\mathbb{E}_{t}^{\mathbb{P}}\left[\left|\boldsymbol{\theta}_{t, S}^{\lambda}(T) \cdot \boldsymbol{Y}(T)-S\right| \cdot I_{\boldsymbol{\theta}_{t, S}^{\lambda}(T) \cdot \boldsymbol{Y}(T)<S}\right] .
\end{gathered}
$$

Proposition 3 reveals that the difference between the expectation of the LAMV hedging portfolio $\mathbb{E}_{t}^{\mathbb{P}}\left[\boldsymbol{\theta}_{t, S}^{\lambda} \cdot \boldsymbol{Y}(T)\right]$ and the claim $\mathbb{E}_{t}^{\mathbb{P}}[S]$ equals that between the expected absolute deviations from gains and losses. As expected, this difference increases with the loss aversion coefficient $\lambda$. 


\subsection{Super- and sub-replicating}

Now, we introduce the super-replicating and sub-replicating cases as well as their $\lambda$ conditions.

Proposition 4 Consider the LAMV hedger $\boldsymbol{\theta}_{t, S}^{\lambda}$.

- $\boldsymbol{\theta}_{t, S}^{\lambda}$ super-replicates any claim $S \in \mathcal{C}_{T}: \operatorname{Pr}\left\{\boldsymbol{\theta}_{t, S}^{\lambda}(T) \cdot \boldsymbol{Y}(T) \geq S\right\}=1$ if and only if $\lambda \rightarrow \infty$;

- $\boldsymbol{\theta}_{t, S}^{\lambda}$ sub-replicates any claim $S \in \mathcal{C}_{T}: \operatorname{Pr}\left\{\boldsymbol{\theta}_{t, S}^{\lambda}(T) \cdot \boldsymbol{Y}(T)<S\right\}=1$ if and only if $\lambda \rightarrow 0$.

Proof: From equation (27), we know that for any $S \in \mathcal{C}_{T}, \boldsymbol{\theta}_{t, S}^{\lambda}$ satisfies

$$
\mathbb{E}_{t}^{\mathbb{P}}\left[\boldsymbol{\theta}_{t, S}^{\lambda}(T) \cdot \boldsymbol{Y}(T)-S\right]=\frac{\lambda-1}{\lambda+1} \mathbb{E}_{t}^{\mathbb{P}}\left[\left|\boldsymbol{\theta}_{t, S}^{\lambda}(T) \cdot \boldsymbol{Y}(T)-S\right|\right] .
$$

(a) For any claim $S \in \mathcal{C}_{T}$, if the LAMV hedger $\boldsymbol{\theta}_{t, S}^{\lambda}(T) \cdot \boldsymbol{Y}(T)$ super-replicates, from equation (28), we know

$$
\frac{\lambda-1}{\lambda+1} \rightarrow 1,
$$

and hence $\lambda \rightarrow \infty$.

(a') If $\lambda \rightarrow \infty$, then $\frac{\lambda-1}{\lambda+1} \rightarrow 1$, and we have

$$
\mathbb{E}_{t}^{\mathbb{P}}\left[\boldsymbol{\theta}_{t, S}^{\lambda}(T) \cdot \boldsymbol{Y}(T)-S\right]=\mathbb{E}_{t}^{\mathbb{P}}\left[\left|\boldsymbol{\theta}_{t, S}^{L A M V}(T) \cdot \boldsymbol{Y}(T)-S\right|\right], \text { for any } S \in \mathcal{C}_{T}
$$

It is easy to find that $\boldsymbol{\theta}_{t, S}^{\lambda}$ super-replicates any claim $S \in \mathcal{C}_{T}$.

(b) If the LAMV hedger $\boldsymbol{\theta}_{t, S}^{\lambda}$ sub-replicates any claim $S \in \mathcal{C}_{T}$, from equation (28), we have

$$
\frac{\lambda-1}{\lambda+1}=-1
$$

which implies $\lambda=0$.

(b') If $\lambda=0$, then we have $\frac{\lambda-1}{\lambda+1} \rightarrow 1$ and

$$
\mathbb{E}_{t}^{\mathbb{P}}\left[\boldsymbol{\theta}_{t, S}^{\lambda}(T) \cdot \boldsymbol{Y}(T)-S\right]=\mathbb{E}_{t}^{\mathbb{P}}\left[\left|\boldsymbol{\theta}_{t, S}^{\lambda}(T) \cdot \boldsymbol{Y}(T)-S\right|\right], \text { for any } S \in \mathcal{C}_{T} .
$$

It is easy to find that $\boldsymbol{\theta}_{t, S}^{\lambda}$ super-replicates any claim $S$.

It is intuitive that a larger $\lambda$ increases the punishment for the loss scenarios and drives the LAMV hedging strategy closer to the super-replicating case, and vice versa. An LAMV hedger with a finite positive $\lambda$ may super-replicate (or sub-replicate) some $S \in \mathcal{C}_{T}$, while the $\lambda$ condition in Proposition 4 corresponds to the case when an LAMV hedger super-replicates (or sub-replicates) any claim $S \in \mathcal{C}_{T}$. 


\section{$5 \quad$ Fair dynamic valuation using LAMV hedging}

In this section, we define the class of LAMVHB valuations and provide a practical application. In Section 5.1, we introduce the LAMVHB $t$ - and dynamic valuation on the basis of the LAMV $t$-hedger. In Sections 5.2 and 5.3, we introduce two numerical examples. In these two examples, we implement the MVHB, LAMV, and LAMVHB dynamic valuations of an equity-linked life insurance contract and a guaranteed minimum accumulation benefit (GMAB) variable annuity, respectively.

\subsection{LAMVHB dynamic valuation}

After having introduced the class of LAMV hedgers and LACHB dynamic valuation in the previous sections, we introduce the notion of dynamic valuation.

Definition 13 (LAMVHB $t$ - and dynamic valuation) Consider a $C H B t$-valuation $\rho_{t}: \mathcal{C}_{T} \rightarrow \mathcal{C}_{t}, t=0,1, \ldots, T-1$ and a $C H B$ dynamic valuation $\left(\rho_{t}\right)_{t=0}^{T-1}$,

- $\rho_{t}$ is an LAMVHB $t$-valuation if there exists an LAMVt-hedger $\theta_{t}$ and a modelconsistent $t$-valuation $\pi_{t}$ such that

$$
\rho_{t}[S]=\boldsymbol{\theta}_{t, S}^{\lambda}(t+1) \cdot \boldsymbol{Y}(t)+\pi_{t}\left[S-\boldsymbol{\theta}_{t, S}^{\lambda}(T) \cdot \boldsymbol{Y}(T)\right], \quad \text { for any } S \in \mathcal{C}_{T}
$$

- $\left(\rho_{t}\right)_{t=0}^{T-1}$ is an LAMVHB dynamic valuation if all $\rho_{t}$ are MVHB $t$-valuations.

The LAMVHB $t$-valuation (dynamic valuation) is a particular class of the LACHB $t$-valuation (dynamic valuation). Hence, according to Corollary 1 , we can prove that the class of LAMVHB valuations is identical to the class of fair valuations.

Corollary 2 Consider a t-valuation $\rho_{t}$ (dynamic valuation $\left.\left(\rho_{t}\right)_{t=0}^{T-1}\right)$. This is an LAMVHB $t$-valuation (dynamic valuation) if and only if it is a fairt-valuation (dynamic valuation).

Hereafter, we show that MVHB $t$-valuation (dynamic valuation), a concept introduced and investigated in previous studies (e.g. Barigou and Dhaene (2019)), as well as LAMV $t$-valuation (dynamic valuation) are two particular classes of LAMVHB $t$-valuation (dynamic valuation).

Definition 14 (MVHB and LAMV $t$ - and dynamic valuation) Consider an LAMVHB $t$-valuation $\rho_{t}$ and an LAMVHB dynamic valuation $\left(\rho_{t}\right)_{t=0}^{T-1}$ determined in equation (29),

- $\rho_{t}\left(\left(\rho_{t}\right)_{t=0}^{T-1}\right)$ is an MVHB t-valuation (dynamic valuation) if the hedger $\boldsymbol{\theta}_{t, S}^{\lambda}$ 's coefficient $\lambda=1$; 
- $\rho_{t}\left(\left(\rho_{t}\right)_{t=0}^{T-1}\right)$ is an LAMV t-valuation (dynamic valuation) if $\pi_{t}[S]=0$ for any $S \in \mathcal{C}_{T}$.

Next, we show that any LAMV convex $t$-valuation (dynamic valuation) is an MVHB $t$-valuation (dynamic valuation).

Corollary 3 Any LAMV t-valuation (dynamic valuation) is an MVHB t-valuation (dynamic valuation).

Proof: Consider the LAMV $t$-hedger $\boldsymbol{\theta}_{t}^{\lambda}$. We prove that $\boldsymbol{\theta}_{t}^{\lambda}$ satisfies the definition of an MVHB $t$-valuation. For any claim $S \in \mathcal{C}_{T}$, we have

$$
\begin{aligned}
\boldsymbol{\theta}_{t, S}^{\lambda}(t+1) & =\arg \min _{\boldsymbol{\mu}_{t} \in \Theta_{t}} \mathbb{E}_{t}^{\mathbb{P}}\left[u\left(\boldsymbol{\mu}_{t}(T) \cdot \boldsymbol{Y}(T)-S\right)\right] \\
& \left.=\boldsymbol{\theta}_{t, S}^{M V}(t+1)+\arg \min _{\boldsymbol{\mu}_{t}^{\prime} \in \Theta_{t}} \mathbb{E}_{t}^{\mathbb{P}}\left[u\left(\left(\boldsymbol{\mu}_{t}^{\prime}(T)+\boldsymbol{\theta}_{t, S}^{M V}(T)\right) \cdot \boldsymbol{Y}(t)-S\right)\right]\right] \\
& =\boldsymbol{\theta}_{t, S}^{M V}(t+1)+\boldsymbol{\theta}_{t, S-\boldsymbol{\theta}_{t, S}^{\lambda V} \cdot \boldsymbol{Y}(t)}^{\lambda V}(t+1) .
\end{aligned}
$$

Then, for any $S \in \mathcal{C}_{T}$, the LAMV $t$-valuation is

$$
\begin{aligned}
\rho_{t}[S] & =\boldsymbol{\theta}_{t, S}^{\lambda}(t+1) \cdot \boldsymbol{Y}(t) \\
& =\boldsymbol{\theta}_{t, S}^{M V}(t+1) \cdot \boldsymbol{Y}(t)+\pi_{t}\left[S-\boldsymbol{\theta}_{t, S}^{M V}(T) \cdot \boldsymbol{Y}(T)\right],
\end{aligned}
$$

where $\pi_{t}[S]=\boldsymbol{\theta}_{t, S}(t+1)^{\lambda} \cdot \boldsymbol{Y}(t)$. Subsequently, we prove that this $\pi_{t}[S]$ is model-consistent.

Consider any $t$-orthogonal $T$-claim $S^{\perp} \in \mathcal{O}_{T}^{t}$. The function $u(x)$ is a convex function. Taking into account the independence of $S^{\perp}$ and $\mathbf{Y}$ as well as Jensen's inequality, we find that for any trading strategy $\boldsymbol{\mu}_{t} \in \Theta_{t}$, any convex function $u(x)$ satisfies

$$
\mathbb{E}_{t}^{\mathbb{P}}\left[u\left(\boldsymbol{\mu}_{t}(T) \cdot \boldsymbol{Y}(T)-S^{\perp}\right) \mid S^{\perp}\right] \geq u\left(\boldsymbol{\mu}_{t}(T) \cdot \mathbb{E}_{t}^{\mathbb{P}}[\boldsymbol{Y}(T)]-S^{\perp}\right) .
$$

Taking the expectations on both sides leads to

$\mathbb{E}_{t}^{\mathbb{P}}\left[u\left(\boldsymbol{\mu}_{t}(T) \cdot \boldsymbol{Y}(T)-S^{\perp}\right)\right] \geq \mathbb{E}_{t}^{\mathbb{P}}\left[u\left(\boldsymbol{\mu}_{t}(T) \cdot \mathbb{E}_{t}^{\mathbb{P}}[\boldsymbol{Y}(T)]-S^{\perp}\right)\right] \geq \mathbb{E}_{t}^{\mathbb{P}}\left[u\left(\widetilde{\rho}_{t}\left[S^{\perp}\right]-S^{\perp}\right)\right]$, which holds for any $\boldsymbol{\mu}_{t} \in \Theta_{t}$. Since $\widetilde{\rho}_{t}\left[S^{\perp}\right]$ can be rewritten as

$$
\widetilde{\rho}_{t}[S]=\left(\rho_{t}\left[S^{\perp}\right], 0, \ldots, 0\right) \cdot \boldsymbol{Y}(T),
$$

with the relation between $\rho_{t}\left[S^{\perp}\right]$ and $\widetilde{\rho_{t}}[S]$ indicated in equation (4) for any $S^{\perp} \in \mathcal{O}_{T}$, we have

$$
\arg \min _{s \in \mathbb{R}} \mathbb{E}_{t}^{\mathbb{P}}\left[u\left(s-S^{\perp}\right)\right]=\mathbb{E}_{t}^{\mathbb{P}}\left(S^{\perp}\right)+\arg \min _{s \in \mathbb{R}} \mathbb{E}_{t}^{\mathbb{P}}\left[u\left(s+\mathbb{E}_{t}^{\mathbb{P}}\left(S^{\perp}\right)-S^{\perp}\right)\right] .
$$

Then, it is straightforward to verify that $\pi_{t}[S]$ is a model-consistent valuation satisfying $\pi_{t}^{L A M V}\left[S^{\perp}\right]=B(t, T) \cdot\left[\mathbb{E}_{t}^{\mathbb{P}}\left(S^{\perp}\right)+\arg \min _{s \in \mathbb{R}} \mathbb{E}_{t}^{\mathbb{P}}\left[u\left(s-\left(S^{\perp}-\mathbb{E}_{t}^{\mathbb{P}}\left(S^{\perp}\right)\right)\right)\right]\right], \quad$ for any $S^{\perp} \in \mathcal{O}_{T}$.

Therefore, we can conclude that the LAMV $t$-valuation $\rho_{t}[S]$ is an MVHB $t$-valuation and that any LAMV dynamic valuation is an MVHB dynamic valuation.

Corollary 3 implies that any LAMV $t$-valuation (dynamic valuation) can be represented in the form of MVHB $t$-valuation (dynamic valuation). In other words, although the LAMV valuation approach does not have an explicit component of the risk margin, it implicitly accounts for the safety margin embedded in the hedging. 


\subsection{Numerical example: Fair dynamic valuation of an equity- linked liability}

In this section, we implement the MVHB, LAMV, and LAMVHB dynamic valuations to valuate an equity-linked life insurance contract. Consider a financial actuarial world in which a zero-coupon bond and a stock are traded. The time- $t$ price of the zero-coupon bond and stock are $B(t, T)$ and $Y^{(1)}(t)$, respectively. The $(t+1)$-value $Y^{(1)}(t+1)$ equals either $Y^{(1)}(t) \cdot u$ or $Y^{(1)}(t) \cdot d$, where $u>1$ and $d<1$. For brevity, in this example, we consider a constant interest rate $r$ such that $B(t, T)=e^{-r(T-t)}$ for $t \leq T$. In this world, we also observe a non-traded survival index $\mathcal{I}(T)$ at time $T$, with its value being either 0 (if few people of a given population survive) or 1 (if many survive).

At any time $t, t=0,1, . ., T-1$, the set of future possible scenarios is

$$
\{(0, d),(1, d),(0, u)(1, u)\},
$$

where each element denotes a possible scenario. The first component of any couple corresponds to a possible value of the survival index $\mathcal{I}$ at time $T$, and $Y^{(1)}(t+1)$ equals $Y^{(1)}(t)$ multiplied by the second component value. $p_{i j}$, which stands for $\mathbb{P}[(i, j)]$ in the real-world probability measure $\mathbb{P}$ is characterized by

$$
p_{0 d}=\frac{1}{6}, p_{1 d}=\frac{1}{6}, p_{0 u}=\frac{2}{6} \text { and } p_{1 u}=\frac{2}{6} .
$$

The time- $T$ survival index value $\mathcal{I}(T)$ and stock price $Y^{(1)}(t+1)$ are mutually independent under the physical measure $\mathbb{P}$ at any time $t, t=0,1, . ., T-1$, implying that the survival index is orthogonal. Further, we have $E^{P}[\mathcal{I}(2)]=\frac{1}{2}$.

We consider a non-traded hybrid claim payable at $T=2$,

$$
S=\mathcal{I}(T) \times \frac{1}{2}\left(Y^{(1)}(1)+Y^{(1)}(2)\right)
$$

whose payoff is equity-linked. That is, the claim $S$ pays survivors the average price of $Y^{(1)}$ at $t=1$ and 2. In the following sections, we implement and compare the MVHB, LAMV, and LAMVHB dynamic valuations. The risk margins of the MVHB and LAMVHB valuations are calculated using the standard deviation risk margin principle:

$$
\pi_{t}[X]=\mathbb{E}_{t}^{\mathbb{P}}[X]+e^{-r} \delta \cdot \sigma_{t}^{\mathbb{P}}\left[X-\mathbb{E}_{t}^{\mathbb{P}}[X]\right], \quad \text { for any } X \in \mathcal{C} .
$$

\subsubsection{MVHB dynamic valuation}

First, we apply the MVHB dynamic valuation approach. At time 1 , the MV hedge of $S$ is given by

$$
\begin{aligned}
\boldsymbol{\theta}_{1, S}^{M V} & =\arg \min _{\boldsymbol{\mu}_{1} \in \Theta_{1}} \mathbb{E}^{\mathbb{P}}\left[\left(\boldsymbol{\mu}_{1} \cdot \boldsymbol{Y}(T)-S\right)^{2}\right] \\
& =E^{P}[\mathcal{I}(2)] \times\left(\frac{Y^{(1)}(1)}{2}, \frac{1}{2}\right) \\
& =\left(\frac{Y^{(1)}(1)}{4}, \frac{1}{4}\right)
\end{aligned}
$$


Then, the time- 1 variance of the remaining risk is given by

$$
S-\boldsymbol{\theta}_{1, S}^{M V} \cdot \boldsymbol{Y}(2)=\left(\mathcal{I}(2)-\mathbb{E}_{t}^{\mathbb{P}}[\mathcal{I}(2)]\right) \times \frac{1}{2}\left(Y^{(1)}(1)+Y^{(1)}(2)\right) .
$$

After some tedious calculations, we have the time-1 variance:

$$
\begin{aligned}
& \operatorname{Var}^{\mathbb{P}}\left[S-\frac{1}{4} Y^{(1)}(1)-\frac{1}{4} Y^{(1)}(2)\right] \\
& =\left[\frac{1}{4} Y^{(1)}(1)\right]^{2} \cdot\left[\frac{2}{3}(1+u)^{2}+\frac{1}{3}(1+d)^{2}\right] .
\end{aligned}
$$

Then, the MVHB value of $S$ at time 1 is

$$
\rho_{1}^{M V H B}[S]=\frac{1}{4} Y^{(1)}(1) \cdot e^{-r}+\frac{1}{4} Y^{(1)}(1)+e^{-r} \delta \sqrt{\frac{1}{6}(1+u)^{2}+\frac{1}{12}(1+d)^{2}} \cdot Y^{(1)}(1) .
$$

Thus, using the time consistency of MVHB dynamic valuation, we have

$$
\begin{aligned}
\rho_{0}^{M V H B}[S] & =\rho_{0}\left[\widetilde{\rho}_{1}^{M V H B}[S]\right] \\
& =\left[\frac{1+e^{-r}}{4}+e^{-r} \delta \sqrt{\frac{1}{6}(1+u)^{2}+\frac{1}{12}(1+d)^{2}}\right] \cdot Y^{(1)}(0) .
\end{aligned}
$$

\subsubsection{LAMVHB dynamic valuation}

Next, we consider applying the LAMVHB dynamic valuation for this product claim. At time 1 , the hedge is given by

$$
\boldsymbol{\theta}_{1, S}^{\lambda}=\frac{\lambda}{\lambda+1} \times\left(\frac{Y^{(1)}(1)}{2}, \frac{1}{2}\right),
$$

determined by solving the optimization in equation (17). As $\lambda>1$, we have $\frac{\lambda}{\lambda+1}>\frac{1}{2}$. Similarly, we calculate the following time-1 variance:

$$
\begin{aligned}
& \operatorname{Var}^{\mathbb{P}}\left[S-\frac{\lambda}{\lambda+1} Y^{(1)}(1)-\frac{\lambda}{\lambda+1} Y^{(1)}(2)\right] \\
& =\frac{1}{(\lambda+1)^{2}}\left(\frac{Y^{(1)}(1)}{2}\right)^{2} \cdot\left[\frac{\lambda^{2}+1}{2} \cdot \frac{2(1+u)^{2}+(1+d)^{2}}{3}-\frac{(1-\lambda)^{2}}{4} \cdot\left(1+\frac{2 u+d}{3}\right)^{2}\right] .
\end{aligned}
$$

Then, considering the cost-of-capital risk margin, the time-1 LAMVHB value is

$$
\begin{aligned}
\rho_{1}^{L A M V H B}[S] & =\frac{\lambda}{\lambda+1} \cdot \frac{1+e^{-r}}{2} \cdot Y^{(1)}(1) \\
& +\frac{e^{-r} \delta}{2(\lambda+1)} \sqrt{\frac{\lambda^{2}+1}{2} \cdot \frac{2(1+u)^{2}+(1+d)^{2}}{3}-\frac{(1-\lambda)^{2}}{4} \cdot\left(1+\frac{2 u+d}{3}\right)^{2}} \cdot Y^{(1)}(1) .
\end{aligned}
$$

Similarly, we have

$$
\begin{aligned}
\rho_{0}^{L A M V H B}[S] & =\frac{\lambda}{\lambda+1} \cdot \frac{1+e^{-r}}{2} \cdot Y^{(1)}(0) \\
& +\frac{e^{-r} \delta}{2(\lambda+1)} \sqrt{\frac{\lambda^{2}+1}{2} \cdot \frac{2(1+u)^{2}+(1+d)^{2}}{3}-\frac{(1-\lambda)^{2}}{4} \cdot\left(1+\frac{2 u+d}{3}\right)^{2}} \cdot Y^{(1)}(0) .
\end{aligned}
$$




\subsubsection{LAMV dynamic valuation}

Based on the LAMVHB dynamic valuations, the time-1 and time-0 values of the LAMV dynamic valuations are

$$
\rho_{1}^{L A M V}[S]=\frac{\lambda}{\lambda+1} \cdot \frac{1+e^{-r}}{2} \cdot Y^{(1)}(1)
$$

and

$$
\rho_{0}^{L A M V}[S]=\frac{\lambda}{\lambda+1} \cdot \frac{1+e^{-r}}{2} \cdot Y^{(1)}(0)
$$

respectively.

From Corollary 3, the LAMV $t$-valuation (dynamic valuation) can be represented in the form of the MVHB $t$-valuation (dynamic valuation). Here, for $t=0$ and 1 , we have

$$
\begin{aligned}
\rho_{t}^{L A M V}[S] & =\boldsymbol{\theta}_{t, S}^{M V} \cdot \mathbf{Y}(t)+\tilde{\pi}_{t}\left[S-\boldsymbol{\theta}_{t, S}^{M V}(T) \cdot \mathbf{Y}(T)\right] \\
& =\boldsymbol{\theta}_{t, S}^{M V} \cdot \mathbf{Y}(t)+\frac{\lambda-1}{2(\lambda+1)} \cdot \frac{1+e^{-r}}{2} \cdot Y^{(1)}(t),
\end{aligned}
$$

where the second term $\frac{\lambda-1}{2(\lambda+1)} \cdot \frac{1+e^{-r}}{2} \cdot Y(t)$ is the safety margin for the remaining risk. As $\lambda>1$, we have

$$
\widetilde{\pi}_{t}\left[S-\boldsymbol{\theta}_{t, S}^{M V}(T) \cdot \mathbf{Y}(T)\right]=\frac{\lambda-1}{2(\lambda+1)} \cdot \frac{1+e^{-r}}{2} \cdot Y^{(1)}(t)>0 .
$$

Since both $\boldsymbol{\theta}_{t, S}^{\lambda}$ and $\boldsymbol{\theta}_{t, S}^{M V}$ are fair, the safety margin $\widetilde{\pi}_{t}[S]=\boldsymbol{\theta}_{t, S}^{\lambda} \cdot \boldsymbol{Y}(t)-\boldsymbol{\theta}_{t, S}^{M V} \cdot \boldsymbol{Y}(t)$ is model-consistent.

This example shows that the LAMV $t$-valuation (dynamic valuation) is an MVHB $t$-valuation (dynamic valuation), with an implicit model-consistent risk margin determined using LAMV hedging. Moreover, from equations (31) and (32), we know that there exists a $\lambda^{E}$ such that $\rho_{0}^{M V H B}[S]=\rho_{0}^{L A M V}[S]$ and $\rho_{1}^{M V H B}[S]=\rho_{1}^{L A M V}[S]$, which is

$$
\lambda^{E}=\frac{\frac{1+e^{-r}}{4}+e^{-r} \delta \sqrt{\frac{1}{6}(1+u)^{2}+\frac{1}{12}(1+d)^{2}}}{\frac{1+e^{-r}}{4}-e^{-r} \delta \sqrt{\frac{1}{6}(1+u)^{2}+\frac{1}{12}(1+d)^{2}}}
$$

As $\lambda^{E}$ is claim-dependent, this example shows that the LAC approach can realize the safety margin of fair valuations.

If we adopt the MV hedge as the 'best hedge' (the benchmark hedge), the remaining risk is the difference between the claim payoff and MV hedge outcome, $S-\boldsymbol{\theta}_{S}^{M V}(T) \cdot \mathbf{Y}(T)$. On this basis, the safety margins of the MVHB and LAMV dynamic valuations are found using the explicit risk margin and implicit 'over-hedging' embedded in the LAC hedging technique, respectively. Moreover, the safety margin of the LAMVHB dynamic valuation relies on both the explicit and the implicit approaches. Table 1 shows the safety margins of these three dynamic valuations, highlighting that the safety margin of the LAMVHB dynamic valuation is obtained using the risk margin as well as the LAC hedging technique. 
Table 2: Safety margins of the MVHB, LAMV, and LAMVHB dynamic valuations.

\begin{tabular}{ccc}
\hline Valuation & Safety Margin & Technique \\
\hline MVHB & $e^{-r} \sqrt{\frac{1}{6}(1+u)^{2}+\frac{1}{12}(1+d)^{2}} \cdot Y^{(1)}(0)$ & Risk margin \\
LAMV & $\frac{\lambda-1}{2(\lambda+1)} \cdot \frac{1+e^{-r}}{2} \cdot Y^{(1)}(0)$ & LAMV hedging \\
LAMVHB & $\frac{e^{-r}}{2(\lambda+1)} \sqrt{\frac{\lambda^{2}+1}{2} \cdot \frac{2(1+u)^{2}+(1+d)^{2}}{3}-\frac{(1-\lambda)^{2}}{4} \cdot\left(1+\frac{2 u+d}{3}\right)^{2}} \cdot Y^{(1)}(0)$ & LAMV hedging + Risk margin \\
\hline
\end{tabular}

\subsection{Numerical example: Fair dynamic valuation of a ratchet GMAB liability}

In this section, we implement the LAMVHB dynamic valuation to determine a fair dynamic valuation of a ratchet GMAB variable annuity. A GMAB variable annuity guarantees the minimum amount received by the annuitant after the accumulation period, protecting the value of the annuity from market fluctuations. The guarantee of the ratchet GMAB is linked to the value of a risky asset and it locks in the historical maximal value before maturity.

\subsubsection{Ratchet GMAB liability}

In this example, we consider a typical ratchet GMAB annuity with a payoff

$$
\text { Payoff }=\mathcal{I}(T) \times \max \left(Y^{(1)}(T), G\right), \text { with } t \leq T,
$$

where the index $\mathcal{I}(T)$ at time $T$ equals 1 if the insured survives and 0 otherwise 6 . At time $T$, the insured, if alive, is credited the greater of the stock value $Y^{(1)}(T)$ and the ratchet guarantee amount $G=\max \left(K, \eta \cdot \max _{t_{i} \leq T-1} Y^{(1)}\left(t_{i}\right)\right)$. The guaranteed amount is given by the higher of a fixed return guarantee $K=e^{r T}$ and the $\eta$ ratio of the highest historically recorded stock value before maturity $T$. Thus, the insurer's portfolio of insurance liabilities has the following form:

$$
S=N(T) \times \max \left(Y^{(1)}(T), G\right)
$$

with $N(t)$ a mortality process counting the number of survivals among an initial population of $l_{x}$ policyholders of age $x$.

We assume that the financial market consists of a risk-free asset $Y^{(0)}(t)=e^{r t}$ and a risky stock index $Y^{(1)}(t), t=0,1, \ldots, T$. To simplify the illustrationd, we assume that the stock follows a geometric Brownian motion:

$$
d Y^{(1)}(t)=Y^{(1)}(t)\left(\mu d t+\sigma d W_{1}(t)\right),
$$

\footnotetext{
${ }^{6}$ Such a GMAB payoff is typical, as discussed, for instance, by Bacinello et al. (2011).

${ }^{7}$ The presented approach can be easily adapted to other stock dynamics such as stochastic volatility and Lévy models.
} 
with the parameters $\mu, \sigma>0$. Moreover, the survival function is then defined by

$$
S_{x}(t):=\mathbb{P}\left(T_{x}>t\right)=\exp \left(-\int_{x}^{x+t} \lambda_{x}(s) d s\right),
$$

where $T_{x}$ is the remaining lifetime of an individual aged $x$ at time 0 . In addition, deaths of individuals are assumed to be independent events conditional on knowing the population mortality 8 . Further, mortality intensity is assumed to be stochastic and it follows the dynamics under $\mathbb{P}$ given by

$$
d \lambda_{x}(t)=c \lambda_{x}(t) d t+\xi d W_{2}(t)
$$

with $c, \xi>0$ and $W_{2}(t)$ a standard Brownian motion, independent of $W_{1}(t)$. If we denote $D(t+1)$ as the number of deaths in year $t+1$, the dynamics of the number of active contracts can be described as a nested binomial process as follows: $N(t+1)=N(t)-$ $D(t+1)$ with $D(t+1) \mid N(t), q_{x+t} \sim \operatorname{Bin}\left(N(t), q_{x+t}\right)$ and the one-year death probability $q_{x+t}$ for this age $x$ population from year $t$ to $t+1$.

After introducing the dynamics of $N(t)$ and $Y^{(1)}(t)$, we assign the parameter setting according to relevant studies. The benchmark parameters for the financial market are $r=0.02, \mu=0.02, \sigma=0.1, \eta=0.8$, and $Y^{(1)}(0)=1$. The mortality parameters $\left(\lambda_{x}(0)=\right.$ $0.0087, c=0.0750, \xi=0.000597)$ follow from Luciano et al. (2017) and correspond to male individuals aged 55 at time 0 in the United Kingdom. We assume there are $l_{x}=1000$ initial contracts at time 0 with a maturity of $T=10$ years. Then, we simulate 10000 sample paths for $N(t)$ and $Y^{(1)}(t)$, for $t=1, \ldots, T$.

\subsubsection{Valuation result}

We implement the MVHB, LAMV, and LAMVHB dynamic valuations on the basis of the simulated scenarios. The detailed simulation and calculation processes are described in the appendix.

Figure 3 compares the evolution of the MVHB, LAMV, and LAMVHB dynamic valuations with a cost-of-capital risk margin in equation (14). Consistent with the Solvency II regulation, we adopt $i_{c o c}=0.06$ and $p=99.5 \%$. The time- $t$ valuation is the expected value of all sample paths, $\mathbb{E}^{\mathbb{P}}\left[\rho_{t}[S]\right]$. We observe an increasing trend of the dynamic valuations over time. Two adverse effects over time, namely the upward trend of the stock and the decreasing value of the non-hedgeable risk margin, jointly shape this curve. In this setting, the impact of the increasing stock value is clearly more pronounced.

Moreover, it is expected that the LAMVHB dynamic valuation $\left(\lambda=1.2, i_{c o c}=0.06\right)$ is higher than the MVHB $\left(\lambda=1.0, i_{c o c}=0.06\right)$ and LAMV dynamic valuations $(\lambda=$ $\left.1.2, i_{c o c}=0.00\right)$. In this parameter setting, the LAMV approach has the lowest valuation outcome. The gap between the LAMVHB and LAMV dynamic valuations illustrates the effect of the risk margin. Similarly, the gap between the LAMVHB and LAMV dynamic

\footnotetext{
${ }^{8}$ See Milevsky et al. (2006) for similar assumptions.
} 
valuations represents the effect of loss aversion (from $\lambda=1.0$ to $\lambda=1.2$ ) under LAMV hedging.

Figure 4 compares the LAMVHB dynamic valuations at time 0 with different loss averse coefficients $\lambda$ (the horizon) and risk margins (different curves). Consistent with our intuition, the LAMVHB dynamic valuation increases both with the loss aversion coefficient $\lambda$ and with the cost-of-capital rate $i_{c o c}$. On the one hand, the LAMV dynamic valuation, a particular LAMVHB dynamic valuation with $i_{c o c}=0.00$, represents the cost of LAMV hedging without adding a risk margin. We observe an increasing trend of the LAMV dynamic valuation with the loss aversion coefficient $\lambda$. This numerical result shows that the expectation of the LAMV $t$-hedging portfolio $\boldsymbol{\theta}_{t, S}^{\lambda}(T) \cdot \boldsymbol{Y}(T)$ increases with the loss aversion coefficient $\lambda$.

On the other hand, the increasing trend of the standard deviation risk margin $(\delta=$ $0.15)$ is faster than that of the cost-of-capital one $(i=0.06)$. This contrast can be explained by that the LAMV hedger's loss aversion reduces the loss deviations, whereas it increases the overall deviations of the remaining risk. Thus, the standard deviation risk margin, which also takes into account the gain deviations, may be unsuitable for application to the 'asymmetrical' remaining risk. Instead, a risk margin that measures one-side tail risk such as the cost-of-capital risk margin is a more reasonable choice.

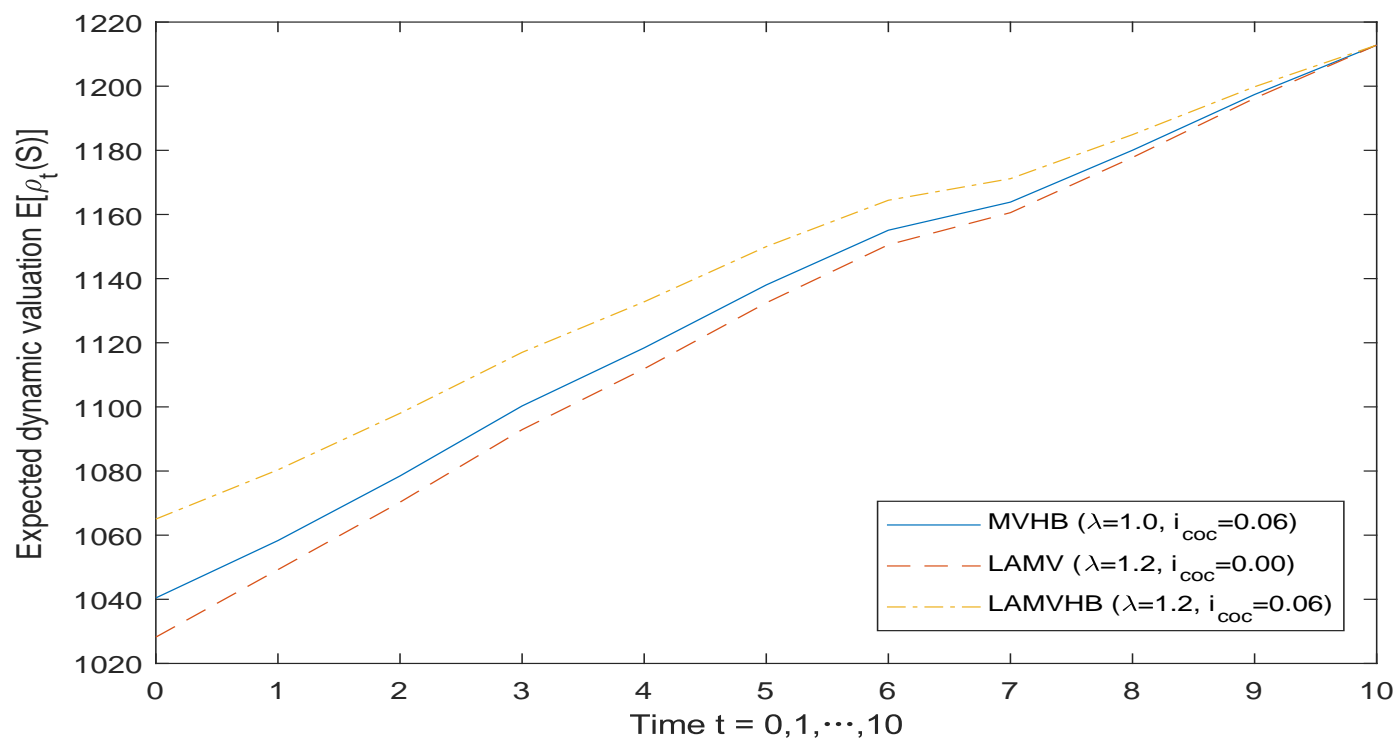

Figure 3: Expected MVHB, LAMV, and LAMVHB dynamic valuations of the GMAB liability. 


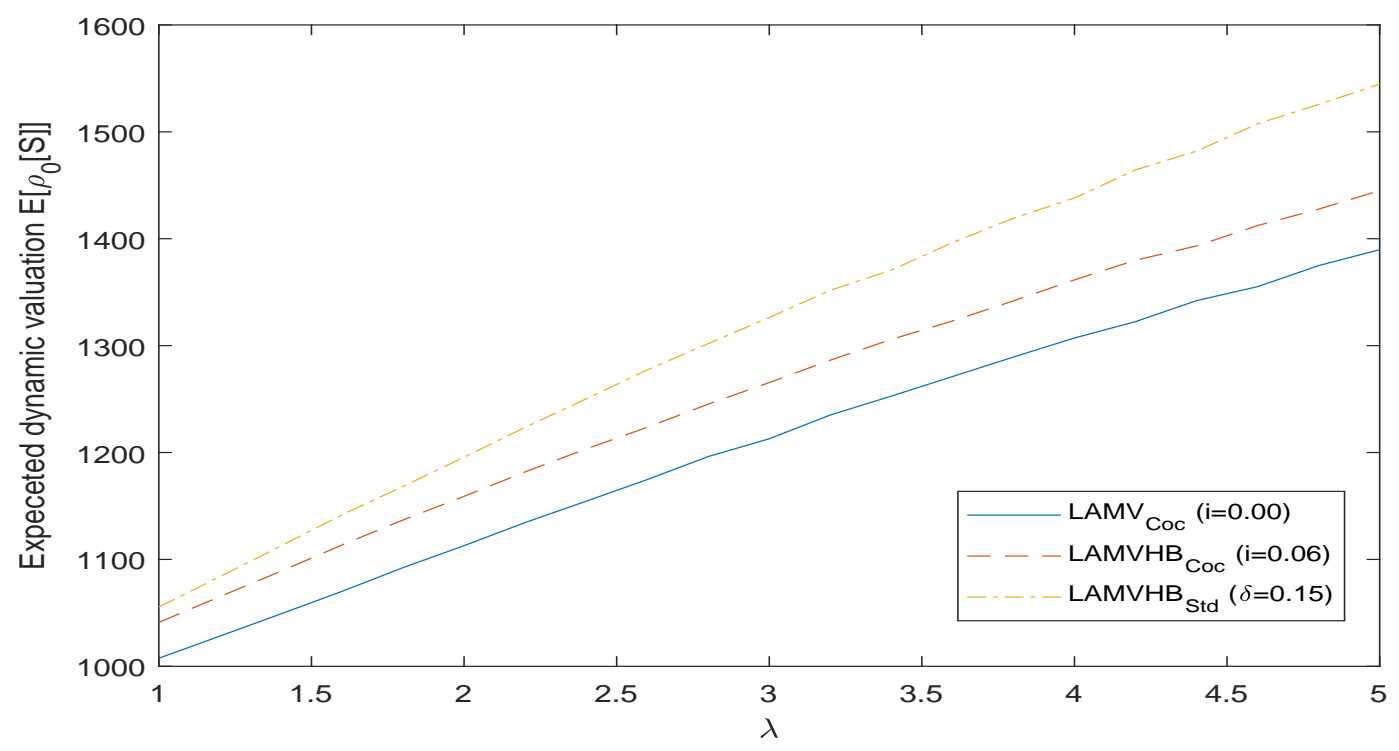

Figure 4: Expected LAMVHB dynamic valuation of the GMAB liability with different cost-of-capital rates.

\section{Conclusions}

Because regulatory directives are ambiguous about how to determine the hedgeable part of a claim, the determination of the fair valuation for insurance liabilities, which are often a combination of hedgeable and unhedgeable risks, is in general not uniquely determined and rather depends on the choice of hedging technique. Since fair dynamic valuation can be implemented using feasible hedging techniques such as convex hedging and MV hedging, the safety margin of fair valuation can also be achieved by hedging in addition to the conventional risk margin approach.

In this study, we investigated the fair dynamic valuation of insurance liabilities using the LAC hedging technique in a multi-period setting. We proved that the classes of LACHB and fair $t$-valuation (dynamic valuation) are equivalent. Moreover, we provided a complete characterization of LAMV hedging and illustrated how LAMV hedging and valuation can be implemented, thus showing that the LAC hedging approach, which provides more conservative hedging, is practical for fair dynamic valuations.

\section{References}

Acciaio, B. and Penner, I. (2011), Dynamic risk measures, in 'Advanced Mathematical Methods for Finance', Springer, pp. 1-34.

Areal, N., Rodrigues, A. and Armada, M. R. (2008), 'On improving the least squares monte carlo option valuation method', Review of Derivatives Research 11(1-2), 119. 
Artzner, P., Delbaen, F., Eber, J.-M., Heath, D. and Ku, H. (2007), 'Coherent multiperiod risk adjusted values and bellman s principle', Annals of Operations Research 152(1), 522 .

Artzner, P. and Eisele, K. (2010), Supervisory accounting: comparison between Solvency 2 and coherent risk measures, in 'Actuarial and Financial Mathematics Conference: Interplay between Finance and Insurance', pp. 3-15.

Bacinello, A. R., Millossovich, P., Olivieri, A. and Pitacco, E. (2011), 'Variable annuities: A unifying valuation approach', Insurance: Mathematics and Economics 49(3), 285297.

Barigou, K., Chen, Z. and Dhaene, J. (2019), 'Fair dynamic valuation of insurance liabilities: Merging actuarial judgement with market-and time-consistency', Insurance: Mathematics and Economics 88, 19-29.

Barigou, K. and Dhaene, J. (2019), 'Fair valuation of insurance liabilities via meanvariance hedging in a multi-period setting', Scandinavian Actuarial Journal (2), 163187.

Bowers, N. L. (1986), Actuarial mathematics, number 517/A18.

Černỳ, A. and Kallsen, J. (2009), 'Hedging by sequential regressions revisited', Mathematical Finance 19(4), 591-617.

Chen, Z., Chen, B. and Dhaene, J. (2019), 'Fair dynamic valuation of insurance liabilities via convex hedging'. Available at SSRN: https://ssrn.com/abstract=3359178.

Cheridito, P. and Kupper, M. (2011), 'Composition of time-consistent dynamic monetary risk measures in discrete time', International Journal of Theoretical and Applied Finance 14(01), 137-162.

Christiansen, M. C. (2013), 'Safety margins for unsystematic biometric risk in life and health insurance', Scandinavian Actuarial Journal pp. 286-323.

Dahl, M. and Møller, T. (2006), 'Valuation and hedging of life insurance liabilities with systematic mortality risk', Insurance: mathematics and economics 39(2), 193-217.

Delbaen, F., Peng, S. and Gianin, E. R. (2010), 'Representation of the penalty term of dynamic concave utilities', Finance and Stochastics 14(3), 449-472.

Delbaen, F. and Schachermayer, W. (2006), The mathematics of arbitrage, Springer Science \& Business Media.

Dhaene, J., Stassen, B., Barigou, K., Linders, D. and Chen, Z. (2017), 'Fair valuation of insurance liabilities: merging actuarial judgement and market-consistency', Insurance: Mathematics and Economics 76, 14-27.

Feinstein, Z. and Rudloff, B. (2015), 'Multi-portfolio time consistency for set-valued convex and coherent risk measures', Finance and Stochastics 19(1), 67-107. 
Föllmer, H. and Schied, A. (2011), Stochastic finance: an introduction in discrete time, Walter de Gruyter.

Frittelli, M. and Gianin, E. R. (2004), 'Dynamic convex risk measures', Risk measures for the 21st century pp. 227-248.

Kaas, R., Goovaerts, M., Dhaene, J. and Denuit, M. (2008), Modern actuarial risk theory: using $R$, Vol. 128, Springer Science \& Business Media.

Kupper, M., Cheridito, P. and Filipovic, D. (2008), Dynamic risk measures, valuations and optimal dividends for insurance, in 'Mini-Workshop: Mathematics of Solvency', Mathematisches Forschungsinstitut Oberwolfach.

Luciano, E., Regis, L. and Vigna, E. (2017), 'Single- and cross-generation natural hedging of longevity and financial risk', Journal of Risk and Insurance 84(3), 961-986.

Malamud, S., Trubowitz, E. and Wüthrich, M. (2008), 'Market consistent pricing of insurance products', ASTIN Bulletin 38(2), 483-526.

Milevsky, M. A., Promislow, S. D. and Young, V. R. (2006), 'Killing the law of large numbers: Mortality risk premiums and the sharpe ratio', Journal of Risk and Insurance $73(4), 673-686$.

Moehr, C. (2011), 'Market-consistent valuation of insurance liabilities by cost of capital', Astin bulletin 41(02), 315-341.

Moreno, M. and Navas, J. F. (2003), 'On the robustness of least-squares monte carlo (lsm) for pricing american derivatives', Review of Derivatives Research 6(2), 107-128.

Newey, W. K. and Powell, J. L. (1987), 'Asymmetric least squares estimation and testing', Econometrica: Journal of the Econometric Society pp. 819-847.

Norberg, R. (2014), 'Life insurance mathematics', Wiley StatsRef: Statistics Reference Online .

Pelsser, A. and Stadje, M. (2014), 'Time-consistent and market-consistent evaluations', Mathematical Finance 24(1), 25-65.

Riedel, F. (2004), 'Dynamic coherent risk measures', Stochastic processes and their applications 112(2), 185-200.

Salzmann, R. and Wüthrich, M. V. (2010), 'Cost-of-capital margin for a general insurance liability runoff', Astin Bulletin 40(02), 415-451.

Schweizer, M. (2010), 'Mean-variance hedging', Encyclopedia of quantitative finance .

Stentoft, L. (2012), American option pricing using simulation and regression: numerical convergence results, in 'Topics in Numerical Methods for Finance', Springer, pp. 57-94.

Thomson, R. J. (2005), 'The pricing of liabilities in an incomplete market using dynamic mean-variance hedging', Insurance: Mathematics and Economics 36(3), 441-455. 
Tsanakas, A., Wüthrich, M. V. and Černỳ, A. (2013), 'Market value margin via meanvariance hedging', Astin Bulletin 43(03), 301-322.

Tversky, A. and Kahneman, D. (1992), 'Advances in prospect theory: Cumulative representation of uncertainty', Journal of Risk and uncertainty 5(4), 297-323.

Wang, Y., Wang, S. and Lai, K. K. (2011), 'Measuring financial risk with generalized asymmetric least squares regression', Applied Soft Computing 11(8), 5793-5800.

Wüthrich, M. V., Merz, M., Wüthrich, M. V. and Wüthrich, M. V. (2013), Financial modeling, actuarial valuation and solvency in insurance, Springer.

Yao, Q. and Tong, H. (1996), 'Asymmetric least squares regression estimation: a nonparametric approach', Journal of Nonparametric Statistics 6(2-3), 273-292.

\section{Appendix}

\section{A1. Simulation and calculation process of the MVHB, LAMV, and LAMVHB $t$ - hedgers}

In this appendix, we introduce the simulation and calculation processes of the MVHB, LAMV, and LAMVHB $t$ - hedgers using a numerical illustration. The procedure is similar to that of Barigou et al. (2019) and Chen et al. (2019).

First, we introduce the generation process of the 10000 simulated paths. For any path $i=1,2, \ldots, 10000$, at any time $t=0,1, \ldots, T-1$ a number of 10000 candidate scenarios of $N_{c}(t+1)$ and $Y_{c}^{(1)}(t+1)$ are generated on the basis of $N(t)$ and $Y^{(1)}(t)$. However, only one scenario is randomly chosen as the simulated $\left(N(t+1), Y^{(1)}(t)\right)$ in path $i$.

Second, at any time $t$ of path $i$, the $t$-hedgers and valuations are based on the 10000 candidate scenarios. At time $t$ of each path, the conditional expected $(t+1)$-valuations are regressed over the risk drivers at time $t+1$ using a second-order least squares regression:

$\mathbb{E}_{t}^{\mathbb{P}}\left[\rho_{t+1}[S] \mid\left(N(t+1), Y^{(1)}(t+1)\right)\right] \approx \beta_{0}+\beta_{1} N(t+1) Y^{(1)}(t+1)+\beta_{2}\left(N(t+1) Y^{(1)}(t+1)\right)^{2}$,

for all scenarios. Here, the choice of the type and number of basis functions is based on an equilibrium between bias and complexity and the payoff structure. The choice of basis functions and their robustness are discussed by Areal et al. (2008), Moreno and Navas (2003), and Stentoft (2012). After having $\beta_{0}, \beta_{1}$, and $\beta_{2}$, we can obtain the 10000 estimated $\rho_{t+1}^{c}[S]$ for all candidate scenarios $\left(N_{c}(t+1), Y_{c}^{(1)}(t+1)\right)$.

On this basis, we apply the LAMV $t$-hedgers. For any path $i$, the hedge at time $t$ is obtained by finding the optimal strategy minimizing the convex punishment function using these 10000 candidate scenarios $\left(N_{c}(t+1), Y_{c}^{(1)}(t+1)\right)$ and estimated $\rho_{t+1}^{c}[S]$. The MVHB $t$-hedger is a particular LAMV $t$-hedger with $\lambda=1$. Finally, the MVHB, LAMV, and LAMVHB values are obtained based on the $t$-hedgers. The expected dynamic valuations of this liability are the expected value of these 10000 simulated scenarios. 